\title{
PLAN DE MARKETING INTERNACIONAL PARA LA EXPORTACIÓN DE DULCES CANUTOS DE ORIGEN COLOMBIANO A CIUDAD AUTÓNOMA DE BUENOS AIRES - CAPITAL FEDERAL - DE LA REPÚBLICA ARGENTINA
}

\author{
Trabajo científico libre la para la obtención del grado de Magister \\ en Marketing Internacional - Facultad de Ciencias Económicas - \\ Universidad Nacional de La Plata.
}

Profesor Director de Tesis:

Prof. Mg. Nestor Aleksink
Presentado por:

David Felipe Ramírez Hernández

Dirección: Calle 159 No 19b - 72 Apto 402

Bogotá - Colombia

Teléfono: (057)3014371159 


\section{PROLOGO}

"Se puede concluir que la memoria humana es un notable sistema afectivo para almacenar y recuperar información"1

José A. Paris

Desde que un ser humano logra tener conciencia y aprende a diferenciar lo bueno de lo malo, el dulce se registra en la memoria como un estimulo físico y mental tan bueno, que logra provocar en el cerebro un cambio en el estado de ánimo de cada persona. Es así como cada cuerpo suele conocer el dulce.

En los países latinoamericanos contamos con una gran variedad de productos en el sector dulcero, encontramos miles de colores, figuras, tamaños, sabores y olores, que siguen cumpliendo con la variada demanda del mercado de las golosinas y productos de confitería. El descontrol de estos productos en los niños a provocado grabes índices de obesidad.

La obesidad es un mal que junto al sedentarismo puede causar graves problemas de salud y para hacer contra a este mal tan común, los médicos y nutricionistas recomiendan una dieta sana, esta ha generado una nueva cultura de alimentación lo que está provocando una demanda creciente de productos orgánicos y naturales.

Es por esta razón que se siguen uniendo más adeptos a la cultura sana y se ve reflejado en la apertura continua de restaurantes de este tipo, que directa o indirectamente trabajan con estos productos, lo que se ve reflejado en la participación del mercado de productos naturales y en este caso Dulces Canutos, lograr más participación en el sector de las golosinas y productos de confitería producidos con frutas de calidad y con un proceso artesanal, lo que nos permite resaltar un nuevo nicho de mercado y poder suplir algunas necesidades de esta nueva cultura, la cultura sana.

Argentina es un país que se destaca en el sector de las golosinas y productos de confitería, las ventas externas de estos productos se han incrementado en el quinquenio $2006-2010$ un $13 \%$, con un crecimiento promedio anual del $4 \%{ }^{2}$,

\footnotetext{
${ }^{1}$ José A. París - Marketing Esencial Un enfoque latinoamericano.

${ }^{2}$ Informe sector alimentos industrializados - Golosinas y Productos de Confitería 2011 - Dirección de Oferta Exportable - Dirección General de Estrategias de Comercio Exterior Subsecretaría de Desarrollo de Inversiones y Promoción Comercial. Ministerio de Relaciones Exteriores y Culto
} 
además la nueva tendencia de specialty foods ${ }^{3}$ o alimentos gourmet tiene más adeptos a ella, gracias a los buenos resultados que se obtienen siguiendo hábitos alimenticios sanos.

Es por eso que estos tres puntos claves; la fortaleza del sector dulcero, las generaciones de nuevas culturas alimenticias y la apertura de establecimientos especializados en alimentación sana hacen que la generación de este proyecto sea muy importante y se considere pasar de lo académico he investigativo a lo práctico comercial en un corto plazo.

\footnotetext{
${ }^{3}$ Programa de apoyo a la reconversión empresarial para las exportaciones módulo mejora de la competitividad sectorial sector alimentos gourmet Diagnóstico del Sector.

ALPHA Estudio de Economía y Negocios S.A. (Agosto de 2006)
} 


\section{ÍNDICE}

Pag.

I. Introducción metodológica

a. Resumen ejecutivo 5

b. Objetivos del proyecto 6

c. Alcance del proyecto 6

$\begin{array}{ll}\text { d. Descripción y filosofía del plan de marketing } & 7\end{array}$

e. Composición Organizacional 8

f. Plan financiero a Largo Plazo 8

II. Análisis y Definición del Mercado Meta

g. Oportunidad comercial entre Colombia y Argentina 10

h. Segmentación de Mercado Meta 11

$\begin{array}{ll}\text { i. Cuantificación de la demanda } & 16\end{array}$

III. Plan de Marketing Internacional (P.E.M.I) para Dulces Canutos

$\begin{array}{ll}\text { j. Estructura Empresarial } & 19\end{array}$

$\begin{array}{ll}\text { k. Unidad de Negocio } & 27\end{array}$

$\begin{array}{ll}\text { I. Posicionamiento } & 27\end{array}$

$\begin{array}{ll}\text { m. Definición del Marketing Mix } & 29\end{array}$

IV. Conclusiones 41

n. Presupuesto y Control 41

o. Análisis financiero $\quad 42$

p. Indicadores financieros; VAN - TIR. 44

q. Punto de equilibrio $\quad 45$

V. Bibliografía 46

Anexos

\section{Tablas}

- Tabla No 1.Concentración de restaurantes de tipo natural por zona

- Tabla No 2. Distribución porcentual de la población por sexo según Centro de Gestión y Participación. Ciudad de Buenos Aires. Año 2004

- Tabla No 3. Distribución porcentual de la población de 25 años o más (1) por máximo nivel de instrucción alcanzado según CGP de la Ciudad Autónoma de Buenos Aires año 2004.

- Tabla No 4. Distribución porcentual de la población de 10 años y más, ocupada, por nivel educativo según Centro de Gestión y Participación. Ciudad de Buenos Aires. Año 2004.

- Tabla No. 5 Cálculo de la demanda Primer Año

- Tabla No. 6 Cálculo de la demanda proyectado a 5 años 
- Tabla No. 7 Posición Arancelaria del Producto a Importar

- Tabla No 8 Los aranceles establecidos

- Tabla No 8.1 Base IVA

- Tabla No.9 Exportaciones colombianas totales - posición arancelaria 1704. A partir del año 2007 y hasta el año 2011 a La Argentina

- Tabla No 10 Simulación de costos de exportación

- Tabla No 11 Matriz de precios y márgenes de ganancia

- Tabla No 12 Cotización de precios sin IVA para la Promoción del Producto

- Tabla No 13 Presupuesto Inicial del Proyecto

\section{Anexos}

- Anexo No 1 mapa económico de C.A.B.A

- Anexo No 2 convenios de comercialización y distribución internacional

- Anexo No 3 ciclos del producto

- Anexo No 4 modelo del pellet con 30 cajas de 22 unidades

- Anexo No 5 modelo pagina web

- Anexo No 6 bruchure promocional

- Anexo No 7 banner promocional

- Anexo No 8 tarjetas de presentación

- Anexo No 9 proyecciones de ventas y costos

- Anexo No 10 flujo de caja proyectado a 5 años 


\section{Introducción Metodológica}

\section{a. Resumen Ejecutivo}

En las diferentes culturas del mundo, más en las latinoamericanas, es muy común hoy en día encontrarnos con productos naturales, orgánicos y vegetarianos a la hora de buscar con que alimentarnos. Verduras, frutas, hortalizas y tubérculos, son productos que se destacan por la forma natural en la que son cultivados ya que nos permiten percibir el sabor original y deleitarnos con platos donde son protagonistas.

El sector de la gastronomía en Ciudad Autónoma de Buenos Aires le está dando cada vez más espacio a la onda natural orgánica y día a día se ven más adeptos a ella gracias a los resultados que muestran las personas que toman el hábito de alimentarse sanamente.

En Ciudad Autónoma de Buenos Aires, es normal encontrar restaurantes de cocina étnica, gourmet, orgánica y natural o de comida saludable en los que se ofrezcan platos de este tipo y sigue creciendo la posibilidad de competir con muchos productos frutales que no se encuentran en Argentina y que puedan exportarse desde Colombia.

Dado este hecho, nace la idea de realizar un análisis investigativo de comercio bilateral entre Colombia y Argentina, teniendo en cuenta fuentes primarias y secundarias de información sobre el sector gastronómico, además de la bibliografía recomendada en el curso para implementar métodos de investigación y ver a fondo los puntos claves que serán tenidos en cuenta a la hora de desarrollar el Plan de Marketing Internacional (PEMI) para un producto como Dulces Canutos. 


\section{b. Objetivos del Proyecto}

Al desarrollar un análisis investigativo de los diferentes factores del mercado, las posibilidades de financiamiento, los factores internos y externos de la empresa y el intercambio comercial de golosinas y productos de confitería que se ha venido presentando entre Colombia y Argentina en los últimos años, podemos afirmar que el principal objetivo es diseñar un Plan de Marketing Internacional (PEMI) para Dulces Canutos de origen Colombiano al mercado Argentino. Este inicialmente es de rigor académico para luego ser llevado a cabo en el mediano plazo al mercado elegido; Ciudad Autónoma de Buenos Aires.

Con el fin de cumplir con el objetivo principal, se aspira a lograr los siguientes objetivos específicos del proyecto.

- Determinar el mercado donde se tiene planeado entrar a competir.

- Segmentación del mercado meta.

- Definir el producto de importación.

- Fijar los costos iniciales del proyecto.

- Establecer el tipo de embalaje y empaque para la comercialización del producto.

- Seleccionar los canales de distribución para la comercialización del producto.

- Planteamiento del plan promocional para los canales de distribución elegidos.

- Estipular el plan logístico para la importación y comercialización.

- Identificar las barreras arancelarias y no arancelarias así como todos los aspectos legales que se deben tener en cuenta para el ingreso del producto.

- Estimar el precio de venta del producto.

- Aclarar la viabilidad financiera del proyecto.

\section{c. Alcance del Proyecto}

El presente proyecto de estudio, se basa en el análisis de varios puntos clave del sector gastronómico de Ciudad Autónoma de Buenos Aires, dándole un enfoque especial a la tendencia de alimentación gourmet, orgánica y naturista (aceptación, hábitos de consumo, competencia local, variedad de gustos o sabores) que se viene dando en C.A.B.A.

El análisis de varios puntos determinantes para el desarrollo del PEMI será apoyado por fuentes primarias y secundarias, además de entrevistas personales con administradores o dueños de restaurantes de este tipo. 
Se tendrá presente la situación actual de la relación comercial entre los dos países (Colombia y Argentina), con una mirada autentica se buscarán las estrategias más adecuadas para adaptarse a la situación.

Evidentemente la variedad en el mercado de confitados de fruta es poca y se ve como una gran oportunidad de negocio a mediano y largo plazo aplicado en el sector gastronómico gourmet.

\section{d. Descripción y filosofía del plan de marketing}

Con el fin de incursionar y adaptarse a los diferentes cambios del mercado en cuanto a la comida sana o natural se refiere, el sector gastronómico argentino, enfocándose en Ciudad Autónoma de Buenos Aires (C.A.B.A) está reuniendo cada vez más adeptos a esta tendencia, que busca cuidar su salud a través de alimentos sanamente producidos, los cuales permiten degustar el sabor original de las frutas, verduras y tubérculos que nos brinda la tierra.

Afirmando sobre los resultados positivos que ha mostrado la cultura de lo sano o natural (el mercado de los productos orgánicos creció a tasas

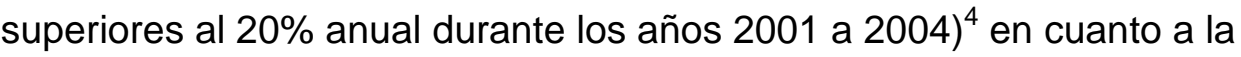
alimentación, y conociendo un tamaño aproximado del mercado de specialty foods o comida Gourmet se refiere, se consolida la idea de importar y comercializar confites de fruta Natural Canutos provenientes de la ciudad de Medellín, Colombia, analizando los factores claves del negocio y cumpliendo con los requerimientos exigidos por los diferentes organismos administrativos de la República Argentina.

"Los nomencladores habituales de actividad económica no identifican específicamente a los productos gourmet. Sin embargo, se puede lograr una aproximación razonable tomando aquellos productos donde la producción gourmet es presumiblemente elevada. Para el año 2005 un valor de USD 43.8 millones de exportaciones y USD 24.9 de importaciones. Es decir, una oferta total (exportaciones más importaciones) de los mencionados USD 70 millones de dólares ${ }^{5 "}$

Actuando dentro del sector gastronómico el proceso de comercialización será en los restaurantes que tengan influencias de cocina orgánica, natural

\footnotetext{
${ }^{4}$ Programa de apoyo a la reconversión empresarial para las exportaciones módulo mejora de la competitividad sectorial sector alimentos gourmet Diagnóstico del Sector. ALPHA Estudio de Economía y Negocios S.A. (Agosto de 2006)

${ }^{5}$ Programa de apoyo a la reconversión empresarial para las exportaciones módulo mejora de la competitividad sectorial sector alimentos gourmet Diagnóstico del Sector.

ALPHA Estudio de Economía y Negocios S.A. (Agosto de 2006)
} 
o gourmet de los sectores analizados de la Ciudad Autónoma de Buenos Aires.

\section{e. Composición Organizacional}

Para la ejecución de este proyecto se establecería una empresa Importadora como S.R.L (sociedad de responsabilidad limitada) la cual tendrá la representación comercial internacional del producto Dulces Canutos.

Inicialmente se requieren tres participantes, cada uno con tareas especificas que logren cumplir con los objetivos establecidos por la empresa:

En primera lugar, un responsable administrativo y contable, en segundo lugar un responsable de marketing y ventas, en tercer lugar un responsable de logística y distribución.

El asesoramiento externo de un tercero para la constitución de la empresa y temas legales es indispensable para la primera etapa de ejecución del proyecto.

Desde Medellín, Colombia, estará el Gerente general de Productos alimenticios Canutos, revisando el proceso de exportación y asegurándose de la calidad y el cumplimiento de envíos.

\section{f. Plan financiero a largo plazo}

El proyecto de importación que se piensa consolidar en la República Argentina, contará con capital propio para consolidar la idea comercial inicialmente en la Ciudad Autónoma de Buenos Aires.

El aporte de capital tendrá una estructura de largo plazo y corresponde a la inversión inicial de los socios.

El aporte será por parte de 2 socios el cual será desembolsado en dos cuotas, en los dos años iniciales de operación, en donde se realizará la conformación de la importadora y el registro de marca y producto.

En los préstamos para emprendimiento, normalmente se cuenta con una asesoría de las entidades estatales para garantizar el éxito de los proyectos, logrando así un reembolso más rápido del préstamo solicitado y ayudando a buscar el éxito. 


\section{Análisis y Definición del Mercado Meta.}

\section{g. Oportunidad comercial entre Colombia y Argentina}

A pesar de los diferentes cambios en la economía mundial durante la última década, al igual que las diferentes crisis económicas y financieras en la zona latinoamericana los dos países que se eligen para el presente proyecto han demostrado un crecimiento notorio en su intercambio comercial gracias a la Asociación Latinoamérica de Integración (A.L.A.D.I) y las buenas relaciones que se han venido presentando entre los dos gobiernos actuales, para acelerar el desarrollo económico y social de los países 6.

Otro punto importante es el intercambio cultural que se ha venido presentando por la gran migración de colombianos a la Republica Argentina y la promoción que realizó la marca país, Colombia es Pasión a través de los diferentes medios publicitarios, generando una gran oportunidad de negocio al notar el vacio que hay en la variedad en confituras de Frutas tropicales sobre el mercado gastronómico argentino.

La calidad y variedad de las frutas tropicales que se encuentra sobre el territorio colombiano, son conocidas en la gastronomía regional, pero debido a las exigencias sanitarias, son pocas las frutas que logran pasar las fronteras nacionales. Viéndolo desde este punto de vista se genera la oportunidad comercial de traer al territorio argentino, una variedad de 7 sabores $100 \%$ naturales (mora, lulo, maracuyá, uchuva, nueces, coco y limón) en forma de confite masticable para iniciar un proceso de apertura a la exportación de la fruta como tal y poder realizar en un futuro la producción en el territorio argentino con las frutas importadas.

El mercado argentino de golosinas y productos de confitería sigue creciendo de forma continua, durante los últimos años el sector se ha consolidado, logrando superar el adverso escenario de 2002. En 2007 la producción superó todos los registros desde $1980^{7}$, además diversas fuentes privadas indican que el $85 \%$ de las confituras azucaradas se comercializan a través de kioscos y mini mercados, el $10 \%$ en comercios tradicionales y un $5 \%$ por medio de supermercados y autoservicios ${ }^{8}$.

\footnotetext{
${ }^{6}$ Aladi - Mercosur, La Intergracion Regional, Hector Froiz Domingo Mazza, Gerardo Woscoboinik

${ }^{7}$ Informe sector alimentos industrializados - Golosinas y Productos de Confitería 2011 - Dirección de Oferta Exportable - Dirección General de Estrategias de Comercio Exterior Subsecretaría de Desarrollo de Inversiones y Promoción Comercial. Ministerio de Relaciones Exteriores y Culto.

${ }^{8}$ Características de la Producción del sector $-{ }^{8}$ Informe sector alimentos industrializados - Golosinas y Productos de Confitería 2011 - Dirección de Oferta Exportable - Dirección General de Estrategias de Comercio Exterior Subsecretaría de Desarrollo de Inversiones y Promoción Comercial. Ministerio de Relaciones Exteriores y Culto.
} 
Como se menciona anteriormente los comercios tradicionales tienen una participación actual del $10 \%$ en el sector, lo que se interpreta como dato clave para la introducción del producto, ya que las grandes empresas se están enfocando en los comercios más populares.

\section{h. Segmentación de Mercado meta}

Reconocido como el mayor aglomerado urbano del país, La Ciudad Autónoma de Buenos Aires, cuenta con 2.859.000 habitantes aproximadamente y una densidad poblacional es de 13.679 habitantes por $\mathrm{km} 2^{9}$, está organizada administrativamente en 16 grupos de participación y gestión (GPG) los cuales son clasificados así:

Zona Norte:

CGP 2 Norte Recoleta

CGP 13: Núñez, Belgrano

CGP 14 Este: Palermo, Villa Crespo

CGP 14 Oeste: Palermo, Belgrano, Colegiales, Chacarita

Zona Sur:

CGP 3: La Boca, Barracas

CGP 4: Boedo, Pompeya, Barracas, San Cristóbal, Parque Patricios

CGP 5: Villa Soldati, Nueva Pompeya

CGP 8: Lugano, Riachuelo, Villa Soldati

Zona Oeste:

CGP 7: Floresta, Villa Luro, Vélez Sarsfield, Flores, Caballito, P. Avellaneda, P. Chacabuco.

CGP 9: Liniers, Mataderos

CGP 10: Versalles, V. Devoto, V. del Parque, Floresta, V. Pueyrredón

CGP 11: Villa Gral. Mitre, Villa Santa Rita, Villa del Parque, Villa Ortúzar, Paternal, Villa Crespo

CGP 12: Coghlan, Saavedra, Belgrano R, Villa Urquiza, Villa Pueyrredón

Zona Este:

CGP 1: Retiro, SanTelmo, Monserrat, San Nicolás, Constitución

CGP 2 Sur: Almagro, Balvanera

CGP 6: Caballito, Almagro, Boedo

\footnotetext{
${ }^{9}$ Programa de Vacancia (PAV 103)- Trabajo, desarrollo y diversidad - Una investigación sobre metodologías y políticas de desarrollo local con acento en la generación de empleo / ingresos Documento final sobre La Ciudad Autónoma de Buenos Aires Cecilia Del Bono-

ceciliadb@uolsinectis.com.ar
} 
Al consultar fuentes oficiales de la ciudad y revisar las zonas donde están los diferentes niveles de "target" económico, detectamos algunas zonas que se destacan por la gastronomía en general como lo veremos en el mapa económico de C.A.B.A (Anexo 1), sin embargo no se especifica claramente el tipo de cocina que se realiza en estas zonas.

Las zonas que se destacan en la parte gastronómica son; Palermo, San Nicolás, Puerto Madero y San Telmo, donde se nota la cercanía a zonas hoteleras reconocidas generando un mercado más amplio contando con clientes locales y extranjeros.

Es complejo encontrar informes de una zona en específico en donde estén ubicados los restaurantes de comida Natural pero gracias a las nuevas herramientas de búsqueda web es posible generar datos del mercado meta y reconocer las zonas donde mayor concentración tiene este tipo de restaurantes.

Al revisar la información en las guías gastronómicas web más populares de Ciudad Autónoma de Buenos Aires, como www. guiaoleo.com.ar, www.restorando.com.ar y www.viaresto.clarin.com, nos encontramos con varios filtros que permiten clasificar los restaurantes por zona, tipo de comida y oscilación de precios.

Al clasificar la información por tipo de cocina NATURAL, se encuentran entre 40 y 50 restaurantes repartidos en las diferentes zonas del perímetro urbano donde se destacan; Palermo, Recoleta, Barrio Norte, Las cañitas, San Telmo, entre otros.

A continuación en la Tabla No 1 veremos la concentración de restaurantes de tipo natural por zona. 
Tabla No 1

\begin{tabular}{|c|c|}
\hline \multicolumn{2}{|c|}{ Concentración de Restaurantes de tipo Natural por Zona. } \\
\hline ZONA & No. DE RESTAURANTE \\
\hline Palermo & 12 \\
\hline San Telmo & 6 \\
\hline Centro & 6 \\
\hline Belgrano & 4 \\
\hline Recoleta & 4 \\
\hline Barrio Norte & 3 \\
\hline Retiro & 3 \\
\hline Las Cañitas & 2 \\
\hline Total & 40 \\
\hline
\end{tabular}

Fuente: Cálculos propios basados en guías gastronómicas web de (C.A.B.A) ${ }^{10}$

Al detallar los informes consultados en donde se abordan variables duras ${ }^{11}$ podemos resaltar los Centros de Gestión y Participación a los que pertenece cada zona definida anteriormente, donde se concentran los restaurantes de cocina Natural en (C.A.B.A).

Al contar con la referencia geográfica de los restaurantes de cocina Natural que más interesan para este proyecto y definidas por centro de gestión y participación se realizaran cálculos de estas, para un análisis más profundo de carácter demográfico.

\section{Distribución porcentual de la población por sexo según Centro de Gestión y Participación. Ciudad de Buenos Aires. Año 2004} (Tabla No. 2)

\begin{tabular}{|c|c|c|c|}
\hline \multirow{2}{*}{$\begin{array}{c}\text { CGP - Centros de } \\
\text { Gestión y Participación. }\end{array}$} & \multicolumn{2}{|c|}{ Sexo } & \multirow{2}{*}{ Total } \\
\cline { 2 - 3 } & Hombres & Mujeres & \\
\hline 1 & 46.3 & 53.7 & 100 \\
\hline 2 Norte & 42.3 & 57.7 & 100 \\
\hline 14 Oeste & 47.3 & 52.7 & 100 \\
\hline 14 Este & 44.2 & 55.8 & 100 \\
\hline
\end{tabular}

Fuente: Clasificación propia basada datos de referencia ${ }^{8}$.

\footnotetext{
${ }^{10}$ www.guiaoleo.com.ar, www.restorando.com, www.viaresto.clarin.com Información tomada el 28 de agosto de 2012 sobre los restaurantes de cocina natural.

${ }^{11}$ Reconocidas como "perfil demográfico" edad sexo nivel socioeconómico, lugar de residencia y nivel educativo.
} 
Como lo refleja la tabla No. 2 el porcentaje de mujeres es más alto en todos los CGP que nos interesan, en primer lugar la CGP 2 Norte con $57.7 \%$ seguido de GCP 14 Este con 55.8\%. Respecto al porcentaje de hombres el CGP 14 Oeste predomina con el 47.3\% seguido del CGP 1 con 46.3\%.

Las mujeres serán quienes más influencien la compra del producto ya que como vemos en la tabla anterior son quienes más presencia tienen en las zonas de interés.

Distribución porcentual de la población de 25 años o más, por máximo nivel de instrucción alcanzado según CGP de la Ciudad Autónoma de Buenos Aires año 2004. (Tabla No. 3)

\begin{tabular}{|c|c|c|c|c|c|c|c|}
\hline \multirow{2}{*}{$\begin{array}{c}\text { CGP - Centros } \\
\text { de Gestión y } \\
\text { Participación. }\end{array}$} & $\begin{array}{c}\text { Hasta } \\
\text { Primario } \\
\text { Imcompleto }\end{array}$ & $\begin{array}{c}\text { Primario } \\
\text { Completo }\end{array}$ & $\begin{array}{c}\text { Secundario } \\
\text { Incompleto }\end{array}$ & $\begin{array}{c}\text { Secundario } \\
\text { Completo }\end{array}$ & $\begin{array}{c}\text { Superior } \\
\text { Universitario } \\
\text { Incompleto }\end{array}$ & $\begin{array}{c}\text { Superior } \\
\text { Universitario } \\
\text { Completo }\end{array}$ & Total \\
\hline 1 & 3.6 & 15.7 & 12.8 & 20 & 17.6 & 30.3 & 100 \\
\hline 2 Norte & 2.3 & 9.6 & 5.6 & 20 & 19.4 & 42.9 & 100 \\
\hline 14 Oeste & 2.2 & 9.9 & 10.2 & 20 & 18.4 & 39.3 & 100 \\
\hline 14 Este & 2.3 & 10.1 & 7.2 & 18 & 18.6 & 43.9 & 100 \\
\hline
\end{tabular}

Fuente: Clasificación propia basada en datos de la referencia ${ }^{8}$.

Dada la distribución porcentual de la tabla No. 3, podemos notar que el CGP 14 Este es el más importante con $43.9 \%$ en nivel superior universitario completo, seguido por muy poco el CGP 2Norte con $42.9 \%$.

El nivel educativo y el grado de ocupación son datos muy importantes para definir el NSE nivel socio económico del mercado sabiendo que la educación, ocupación y patrimonio son los tres variables contempladas para la definición de este ${ }^{12}$.

Según los análisis en la investigación de campo se puede definir que en la cultura argentina en estos CGP los habitantes regularmente salen a los restaurantes de la zona o piden un servicio a domicilio. Además les gusta estar seguros de lo que están comiendo y prefieren restaurantes conocidos donde regularmente asisten y recomiendan a sus amigos y conocidos asistir.

${ }^{12}$ Estandarización Nivel Socioeconómico. Presentación expuesta por el Dr. Gustavo E. González. 
Distribución porcentual de la población con más de 10 años, ocupada, por nivel educativo según Centro de Gestión y Participación.

Ciudad de Buenos Aires. Año 2004. (Tabla No.4)

\begin{tabular}{|c|c|c|c|c|c|c|c|c|}
\hline $\begin{array}{c}\text { CGP - Centros } \\
\text { de Gestión y } \\
\text { Participación. }\end{array}$ & $\begin{array}{c}\text { Primario } \\
\text { Imcompleto }\end{array}$ & $\begin{array}{c}\text { Primario } \\
\text { Completo }\end{array}$ & $\begin{array}{c}\text { Secundario } \\
\text { Incompleto }\end{array}$ & $\begin{array}{c}\text { Secundario } \\
\text { Completo }\end{array}$ & $\begin{array}{c}\text { Superior } \\
\text { Incompleto }\end{array}$ & $\begin{array}{c}\text { Superior } \\
\text { Completo }\end{array}$ & $\begin{array}{c}\text { Sin } \\
\text { Instrucció }\end{array}$ & Total \\
\hline $\mathbf{1}$ & 3.8 & 12.9 & 12.3 & 16.3 & 24.3 & 30.3 & 0.1 & 100 \\
\hline 2 Norte & 2.5 & 7.2 & 5.0 & 14.7 & 24.2 & 46.5 & 0 & 100 \\
\hline 14 Oeste & 1.6 & 4.9 & 9.7 & 15.6 & 26.2 & 41.9 & 0 & 100 \\
\hline 14 Este & 1.5 & 5.8 & 8.7 & 13.7 & 25.3 & 45.1 & 0 & 100 \\
\hline
\end{tabular}

Nota: el nivel educativo superior comprende universitario, terciario y posgrado.

Fuente: Clasificación propia basada en datos de la referencia ${ }^{8 .}$

Al detallar la tabla No 4 se nota la ventaja del CGP 2 Norte con $46.5 \%$ del superior completo ocupada, seguida de 45.1\% del CGP 14 Este. Aquí hay una similitud con los datos anteriores donde podemos concluir que en estos CGP es donde más porcentaje de ocupación hay en el nivel superior de educación alcanzado identificándolos como foco principal y los CGP 1 y 1 Oeste como secundarios en donde también están ubicados algunos restaurantes de cocina natural.

Al definir el mercado que se quiere llegar podemos analizar a qué nivel socio económico pertenece, según lo publicado anteriormente. Por eso la investigación se apoya en la tabla siguiente, cuyos datos fueron extraídos de la fuente citada al pie de la misma. Que nos muestra el porcentaje de la población que pertenece a cada NSE.

\begin{tabular}{|c|c|c|c|}
\hline \multicolumn{4}{|c|}{ DISTRIBUCIÓN DEL ÍNDICE NES-IMAR } \\
\hline Posición & Segmento & $\%$ & \\
\hline $\begin{array}{l}\text { Alto - alto } \\
\text { Alto } \\
\text { Medio alto } \\
\text { Medio bajo } \\
\text { Bajo } \\
\text { Bajo-bajo }\end{array}$ & $\begin{array}{c}\text { A-B-C1 } \\
\text { C2 } \\
\text { C3 } \\
\text { D1 } \\
\text { D2 } \\
\text { E } \\
\end{array}$ & $\begin{array}{c}7 \\
11 \\
26 \\
22 \\
29 \\
5 \\
\end{array}$ & $\begin{array}{c}\text { afluentes } \\
\text { clase media } \\
\text { clases bajas }\end{array}$ \\
\hline & & 100 & \\
\hline
\end{tabular}

Fuente: Ipsos-Mora y Araujo/Analogias-RIOP, 2001.

Fuente: http://www.eclac.cl/publicaciones/xml/7/11307/L1772e-P.pdf. Información tomada el 30 de agosto del 2012.

Se entiende que nuestros clientes finales son los comensales de cada restaurante pero se genera una cadena con los administradores, encargados y gerentes de compras que buscan dar un valor agregado a su restaurante través de un obsequio único en el momento final del servicio, en esta ocasión serán los Dulces Canutos.

Definiendo nuestro target como perteneciente al segmento C3, C2, A-B-C 1 o a la posición media alta, alta y alta alta, podemos realizar la creación de 
un perfil de consumidor final del producto Dulces Canutos ubicado en el mercado objetivo definido.

Teniendo en cuenta que los consumidores finales del producto son los comensales de cada uno de los restaurantes de cocina natural, hablamos de hombres y mujeres entre 25 y 60 años ubicados en los CGP 1, 2, 14 Oeste y 14 Este pertenecientes al segmento C3, C2, A-B-C 1 de la Ciudad Autónoma de Buenos Aires los cuales buscan cuidar su salud con una alimentación sana y acuden a restaurantes de cocina natural conocidos donde están seguros de la preparación e ingredientes utilizados.

Tienen un nivel de educación superior y regularmente salen a cenar con un amigo, pareja o con la familia. Sabe diferenciar los alimentos que más lo nutren y alguna vez ha sido asesorado por un nutricionista.

\section{i. Cuantificación de la Demanda}

En este punto se realizó una investigación donde el tipo de muestra es no probabilístico, en donde la muestra fue tomada bajo el mismo criterio de conveniencia en la segmentación del mercado, donde se eligieron dos restaurantes de cada zona identificada como mercado objetivo (CGP 1, 2, 14 Oeste y 14 Este). En total fueron 10 de un mercado de 40 restaurantes de cocina natural a los cuales se acudió para solicitar una opinión objetiva en donde la conversación fue guiada a la tendencia de alimentación sana y a una crítica constructiva del producto y su presentación por parte de los chef's, encargados o administradores, después de probar Dulces Canutos.

En el trabajo de campo se realizo un conteo de mesas y sillas de cada restaurante, se cálculo un promedio de rotación de cubiertos por día de 60 personas como mínimo promedio en los restaurantes de cocina natural, en donde se realizó una ponderación para estimar una demanda de 20 restaurantes en el primer trimestre y 35 restaurantes al finalizar el cuarto trimestre como meta, logrando una buena aceptación del producto, teniendo en cuenta la apertura de nuevos restaurantes.

Se tuvo en cuenta el número de cubiertos mínimo por día (60) en cada restaurante para estimar la cantidad de producto (paquetes $\times 200$ unidades multi sabores) que se requieren por mes y trimestre para calcular el pedido anual en paquetes de 200 unidades. 
Tabla No. 5 Cálculo de la demanda Primer Año.

\begin{tabular}{|l|c|c|c|c|c|}
\hline & \# Restaurante & $\begin{array}{c}\text { Cubiertos Mes x } \\
\text { Restaurante }\end{array}$ & $\begin{array}{c}\text { Paquetes de 200 } \\
\text { Unidades } \mathbf{x} \text { Restaurante }\end{array}$ & $\begin{array}{c}\text { Paquetes de 200unidades } \\
\mathbf{2} \text { 20 restaurantes al mes }\end{array}$ & $\begin{array}{c}\text { Pedido trimestral } \mathbf{2 0} \\
\text { restaurantes en } \\
\text { paquetes de 200 und }\end{array}$ \\
\hline Primer trimestre & 20 & 1800 & 9 & 180 & 540 \\
\hline Segundo trimestre & 25 & 1800 & 9 & 225 & 675 \\
\hline Tercer trimestre & 30 & 1800 & 9 & 270 & 810 \\
\hline Cuarto trimestre & 35 & 1800 & 9 & 315 & 945 \\
\hline \multicolumn{7}{|r}{} & $\begin{array}{c}\text { Total de Paqutes x 200 } \\
\text { unidades Primer año }\end{array}$ & $\mathbf{2 9 7 0}$ \\
\cline { 3 - 5 }
\end{tabular}

Fuente: Cálculos propios

En la tabla No 5 se define que son 2.970 paquetes de 200 unidades como pedido del primer año teniendo en cuenta que se plantea una meta del $25 \%$ de crecimiento trimestral en la cartera de clientes, sobre la base inicial.

Es importante resaltar que la demanda puede aumentar o disminuir según las estaciones del año siendo la primavera y el otoño los picos de venta y verano e invierno disminución de ventas a lo que se reaccionará con una estrategia a mencionar en capítulos posteriores.

Tabla No. 6 Cálculo de la demanda proyectado a 5 años

\begin{tabular}{|c|r|r|r|r|}
\hline \multicolumn{5}{|c|}{ Numero de Paquetes x 200 Unidades } \\
\hline Primer año 2013 & Segundo año 2014 & Tercer año 2015 & Cuarto año 2016 & Quinto año 2017 \\
\hline 2970 & 4.604 & 7.135 & 11.060 & 17.143 \\
\hline
\end{tabular}

Fuente: Cálculos propios

Como se puede ver en la tabla No 6 el crecimiento anual es de $55 \%$ en el segundo año, en el tercer año el crecimiento es de 65\% y para el quinto año de $82 \%$ teniendo en cuenta el primer año, así logrando en el quinto año 17.143 paquetes $x 200$ unidades, lo que hace positivo el panorama del proyecto, cabe anotar que solo se estimará la venta de un solo producto para la proyección hecha en la tabla No 6 ,teniendo en cuenta que logrando resultados positivos se ampliará la variedad de productos importados.

Se estima que para mediados del segundo año se esté completando la exportación de otro producto como puede ser; mermeladas o choco canutos lo que haría más fuerte la consolidación del proyecto. 


\section{Plan de Marketing Internacional (P.E.M.I) para Dulces Canutos}

\section{j. Estructura empresarial}

\section{Análisis del Ambiente Externo Internacional}

Aquí podemos resaltar las diferentes estrategias que han implementado los gobiernos regionales para el desarrollo de la economía como por ejemplo el Acuerdo de Complementación Económica ACE No 59 donde en el marco jurídico de la Asociación Latinoamericana de Integración ALADI se firmó este acuerdo entre los países que hacen parte de MERCOSUR y Colombia, Ecuador y Venezuela, donde a partir de los primeros días del año 2005 entró en vigencia.

Este acuerdo busca formar un Área de Libre Comercio entre las partes contratantes mediante la expansión y diversificación del intercambio comercial y la eliminación de las restricciones arancelarias y de las noarancelarias que afecten al comercio recíproco ${ }^{13}$.

Dentro del acuerdo de Complementación Económica No. 59 se estableció un Programa de Liberación Comercial que permite el desarrollo de la Zona de Libre Comercio, este se aplica a los productos originarios y procedentes de los territorios de las partes Firmantes, desgravando aranceles de forma anual y progresiva.

En la tabla que se verá a continuación encontraremos la posición arancelaria en la cual está enmarcado el producto a Importar.

\footnotetext{
${ }^{13}$ Secretaria General de la comunidad andina, documentos de información, http://intranet.comunidadandina.org/Documentos/DInformativos/SGdi671.pdf información tomada el 25 de agosto de 2012.
} 
Tabla No. 7 Posición Arancelaria del Producto a Importar

\begin{tabular}{|c|l|}
\hline \multicolumn{2}{|l|}{ CLASIFICACIÓN ARANCELARIA } \\
\hline Descripcion & $\begin{array}{l}\text { Productos de las industrias alimentarias; bebidas, líquidos alcohólicos y } \\
\text { vinagre; tabaco y sucedáneos del tabaco, elaborados }\end{array}$ \\
\hline Capítulo 17 & Productos a base de gelificantes, incluidas las pastas de frutas \\
\hline Partida 1704 & Artículos de confitería sin cacao (incluido el chocolate blanco). \\
\hline Sistema Amortizado 1704.90. & Los Demas \\
\hline $\mathbf{1 7 0 4 . 9 0 . 2 0 . 4 9 9 W}$ & Los Demas \\
\hline
\end{tabular}

Fuente: AIERA (Asociación de importadores y exportadores de la republica argentina)

Nota: En principio, por la cantidad de fruta serían clasificados en el capítulo 20, pero hay una nota de exclusión para cuando se presentan como artículos de confitería de consumo inmediato14.

\section{Los aranceles establecidos para la anterior partida expuesta en la Tabla No 8 son los siguientes:}

\begin{tabular}{|l|r|}
\hline \multicolumn{2}{|c|}{ Aranceles Partida 170.90.20.499w } \\
\hline Derechos de Importación & $20 \%$ \\
\hline Tasa Estadística* & $0.0 \%$ \\
\hline
\end{tabular}

Fuente: AIERA.

*La tasa estadística quedaría Exenta, Únicamente Presentando Certificado de origen del producto, por convenio ALADI.

Tabla No 8.1 Base IVA

\begin{tabular}{|l|r|}
\hline IVA Adicional & $21 \%$ \\
\hline IVA & 10,5 \\
\hline Ganancias & $3 \%$ \\
\hline Ingresos Brutos & $1,50 \%$ \\
\hline
\end{tabular}

Los Ítems de la tabla anterior no se tendrán en cuenta como costos para el cálculo de Flujo de caja ya que corresponden al crédito fiscal que se reintegra con las ventas.

En la tabla anterior se determinan los aranceles e impuestos que se abonan para el correcto proceso de importación en Argentina de dulces Canutos, uno de ellos con preferencias arancelarias como se menciona en el punto anterior en lo que refiere a derechos de importación es de $20 \%$ sobre el valor CIF costo, seguro y flete.

${ }^{14}$ AIERA (asociación de importadores y exportadores de la republica argentina) www.aiera.org.ar 
Continuando con los impuestos se debe tener en cuenta un 0,5\% de Tasa estadística del valor FCA (franco transportista en lugar convenido), que es aplicable a la gran mayoría de productos exceptuando los combustibles, armas, libros, periódicos y bienes de capital no usados entre otros. Como se menciona el producto quedaría exento de este impuesto presentando el Certificado de origen.

Continuamos con el IVA de 21\% decretado por Argentina, en donde sólo algunos productos agrícolas, mineros, educacionales y médicos pagan una tasa diferencial del IVA.

Un IVA adicional del 10,5\%, seguido de impuestos a las ganancias del 3\% y por último un $1.5 \%$ sobre los ingresos brutos.

\section{Regulaciones y normas}

Las regulaciones y normas por las que debe pasar el producto a importar Dulces Canutos en Argentina acompañada de la DJAI (declaración jurada anticipada de importación), son las siguientes:

\section{Intervenciones}

- Alimentos Acondicionados - INAL (instituto nacional de alimentos)

- SENASA - Servicio Nacional de Sanidad y Calidad Agroalimentaria

\section{Licencia}

LAPI = Licencia Automática Previa de Importación

\section{Valoración}

- Declaración de Valor en Aduana

- Alerta de destinaciones Oficializadas

\section{Requisitos}

Embalajes de madera.

Datos oficiales señalan que la economía colombiana respecto a las exportaciones está mostrando datos positivos, entre Enero y Marzo de 2012, se exportaron desde Colombia hacia Argentina 149.603 toneladas, que correspondieron a USD 47.716.897 en valor $\mathrm{FOB}^{15}$.

Las vías de acceso más utilizadas son marítimas y aéreas, con un porcentaje del $98.88 \%$ del total exportado a la fecha la carga marítima es la más común.

\footnotetext{
${ }^{15}$ Proexport Colombia, Promoción de Turismo Inversión y Exportación http://www.proexport.com.co información tomada el 1 de septiembre del 2012.
} 
Tabla No.9 Exportaciones colombianas totales - posición arancelaria 1704. A partir del año 2007 y hasta el año 2011 a La Argentina.

\begin{tabular}{|c|c|c|c|c|c|c|c|}
\hline Capitulo del & Posición & \multirow{2}{*}{ Descripción } & \multicolumn{5}{|c|}{ Miles de Dolares FOB } \\
\cline { 5 - 8 } \\
arancel & Arancelaria & 2007 & 2008 & 2009 & 2010 & 2011 \\
\hline $\begin{array}{c}\text { Azúcares y } \\
\text { articulos de } \\
\text { confiteria }\end{array}$ & 1704 & $\begin{array}{c}\text { Los demas articulos } \\
\text { de confiteria sin } \\
\text { cacao (incluido el } \\
\text { chocolate blanco) }\end{array}$ & 9.520 .023 & 10.633 .178 & 9.493 .688 & 9.012 .955 & 11.251 .197 \\
\hline
\end{tabular}

Fuente: http://www.dane.gov.co información tomada el 21 de agosto del 2012.

Realizando el análisis correspondiente de la tabla No 9 podemos afirmar que la iniciativa por la exportación de este tipo de productos viene creciendo tal y como se nota entre al año 2007 y 2011 se ha incrementado en un $18 \%$ de forma regular pero no tan acelerada como se ve con otro tipo de productos de exportación regular como el café de Colombia y demás.

La estructura logística argentina muestra un buen desempeño recibiendo una calificación actual por parte de la Logistics Performance Index (LPI) ${ }^{16}$ del 3.05 puntos (siendo 1 la calificación más baja y 5 la más alta).

Para el presente proyecto se tiene contemplado acceder por vía aérea, actualmente los servicios aéreos procedentes de Colombia salen desde las siguientes ciudades; Bogotá, Cali y Medellín, lugar de origen de los Dulces Canutos beneficiando la labor logística en el proceso de exportación.

El tipo de cambio es un factor importante, la relación peso argentino frente al dólar, se encuentra actualmente en $\$ 4,65$. Generalmente las variaciones de la cotización afectarían al proyecto de forma directa por que los pagos al fabricante de Dulces Canutos se realizarían en dólares con un plazo mínimo de 30 días.

Si nos fijamos en el mercado gastronómico natural de C.A.B.A podemos resaltar que se identifica una ventaja competitiva en el enfoque de nicho ${ }^{17}$ para el proyecto, resaltando que inicialmente se incursionará en restaurantes de comida natural.

16

http://lpisurvey.worldbank.org/international/scorecard/radar/254/C/ARG/2012/C/ARG/2010/C/ARG/20 07 ? sort=asc\&order=Customs\#datatable información tomada el 30 de agosto de 2012

${ }^{17}$ Warren Keegan, Marketing Globla, Quinta Edición Prents Hall, Madrid 1997 
Teniendo en cuenta que la demanda incipiente es cuando no se tiene conciencia completa de la necesidad del posible cliente; sin embargo puede reconocerse una tendencia positiva que señala a futuro.

Se puede asegurar que en el mercado gastronómico de cocina natural en C.A.B.A se presenta demanda incipiente ${ }^{18}$, otro motivo para iniciar con el proceso de exportación.

\section{Ambiente interno de la empresa}

La creación de la una representación comercial en C.A.B.A, en este caso Importadora Huerta Co S.R.L, trabajará bajo los mismos principios de la empresa fabricante Productos Alimenticios Canutos, de Medellín, Colombia

1. La calidad (los desarrollos buscan calidad antes que precio)

2. De frutas (todos los productos son a base de frutas)

3. Naturales (No utilizan colorantes, ni saborizantes, ni conservantes, ni nada artificial)

4. Proceso ecológico (la casa es un vivero y reprocesa gran parte de los desperdicios orgánicos para producir compost - abono)

5. Desarrollo humano del capital de trabajo (para Canutos la empresa no es solo dinero, sino que es un estilo de vida que trata de inculcar en sus trabajadores y así busca mejorar su nivel de vida y educativo)

La empresa fabricante tiene 10 años en el mercado colombiano, cuenta con los debidos registros de marca trabajando de acuerdo a las BPM (buenas practicas de manufactura) $^{19}$, además actualmente cuenta con una capacidad de producción de 3 toneladas (600.000 Canutos) haciéndola más competitiva y expandiéndose inicialmente en la región sudamericana.

A pesar de que es una receta casera preparada artesanalmente, el proceso de la materia prima se hace con maquinaria que agiliza la labor de despulpar la fruta siendo esta la tecnología aplicada en el proceso de fabricación. La fábrica actualmente cuenta con un espacio amplio donde se

\footnotetext{
${ }^{18}$ Warren Keegan, Marketing Globla, Quinta Edición Prents Hall, Madrid 1997

${ }^{19}$ En Colombia, las buenas prácticas de manufactura (BPM) para alimentos están reguladas por el INVIMA (instituto nacional de vigilancia de medicamentos y alimentos).
} 
puede instalar maquinaria adicional y lograr una capacidad productiva más alta de forma ágil.

Al notar una creciente aceptación por el mercado colombiano durante los 10 años de trabajo, Dulces Canutos busca la internacionalización en el mercado de Argentina a donde se iniciará el proceso de importación como un plan a largo plazo para la consolidación de importadora de alimentos preparados de frutas tropicales y otras variedades de alimentos sanos.

Para la conformación de la empresa es necesario contar con algunos recursos fundamentales guiados a lograr los objetivos planteados.

\section{Recursos Humanos:}

La empresa fabricante actualmente cuenta con una estructura básica de bajo reconocimiento formal, con este proyecto implementará un departamento de comercio exterior operando en Argentina por la empresa Huerta Co S.R.L importadora que se establecerá en C.A.B.A donde se iniciaran operaciones comerciales entre Colombia y Argentina.

\section{Estructura Organizacional - Productos Alimenticios Canutos}

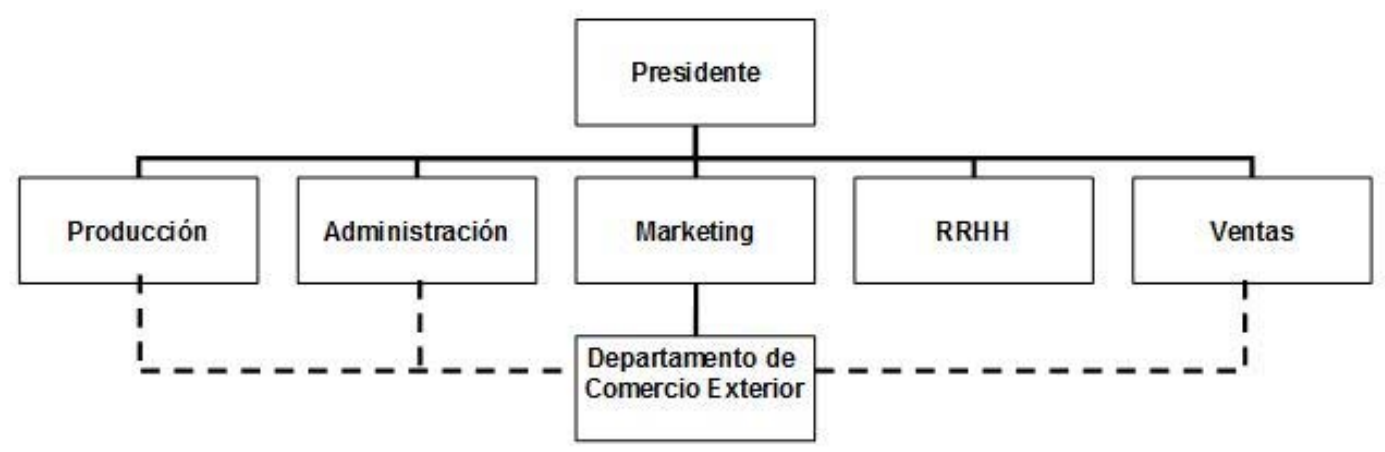

Como se menciona en el primer capítulo, en la composición organizacional de la empresa S.R.L ubicada en C.A.B.A estará dispuesta por tres integrantes; administrativo- contable, marketing - ventas, logística distribución. 


\section{Estructura Organizacional}

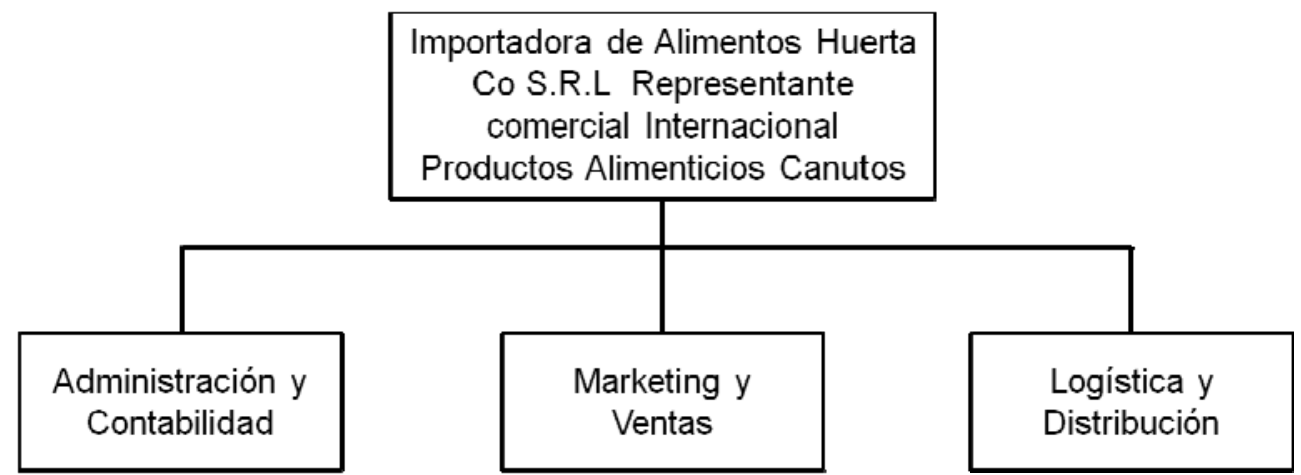

Se deberá realizar un contrato con el fabricante para dejar en claro todos los términos de responsabilidad de ambas partes, para tal motivo se presenta el anexo No 2 con el contrato de representación comercial internacional.

Como se menciona en el primer capítulo se deberá contar con la asesoría externa para la composición de la empresa de sociedad responsable limitada S.R.L , esta obtendrá un Código Único de Identificación Tributaria CUIT con el cual deberá inscribirse ante la Administración Federal de Impuestos Públicos AFIP como importador y exportador para poder empezar a trabajar.

Antes de iniciar labores se debe haber tramitado el registro de marca el cual estará a cargo del Fabricante, el registro de producto del que estará encargada la importadora pero reembolsado por el fabricante con producto, el registro nacional de productos alimenticios R.N.P.A y el registro nacional de establecimientos. (RNE)

Los integrantes de la empresa tendrán asignadas sus tareas y serán responsables de cumplir los objetivos bajo los principios establecidos por la empresa fabricante.

\section{Administración y contabilidad:}

Estará encargado de gestionar todas las tareas relacionadas a la compra de; infraestructura para la empresa, computadores, instrumentos de oficina y pagos a terceros. Además será capacitado por el asesor externo sobre los temas legales de conformación de la empresa, registros de marca y de producto. 


\section{Marketing y Ventas:}

Este colaborador realizara las investigaciones previas para la llegada de cualquier producto que se defina importar, contacto con despachante de aduana, coordinación con el área logística y distribución para la llegada de mercadería, diseño de estrategias de mercadeo, gestión de diseño grafico y campaña publicitaria, visitas organizadas a clientes potenciales, conformación de base de datos y ventas directas.

\section{Distribución y Logística:}

Será el encargado de realizar un seguimiento a las tareas más importante y es la gestión y coordinación para que el producto llegue en los tiempos pactados por el encargado de marketing y ventas con el cliente. Estará atento de la recepción de mercadería en aduana por lo que permanecerá en contacto con el despachante encargado, coordinará con la empresa fabricante las fechas de envió, con el transportador local el movimiento de la mercadería y coordinación del almacenaje de mercadería en un lugar previamente adecuado. Realizara los recorridos de entrega en el mes inicial con la empresa transportadora.

Requerimientos de infraestructura técnica:

- Oficina de operaciones (estratégicamente ubicada según mercado objetivo)

- Dos (2) computadoras portátiles

- Materiales de oficina (resmas de papel, agendas, impresora etc.)

- Estructura física para 5 personas (cubículos con sillas).

- Línea telefónica y fax

- Internet

\section{k. Unidad de Negocio}

Al haber puesto en claro la información sobre las variables que influyen y benefician a la empresa, se define la importación de dulces canutos inicialmente al mercado de C.A.B.A en los restaurantes de cocina natural, se opta por elegir una estrategia de Importación directa ${ }^{20}$ en donde la participación en el proceso aduanero local estará bajo la supervisión del encargado de logística.

Como se menciona en capítulos anteriores esta primera importación será la apertura a la diversificación de los productos fabricados por Productos

\footnotetext{
${ }^{20}$ Presentación - Marketing Internacional en mercados Internacionales - Universidad Nacional de La Plata - Profesora Dra. Cristina Zapata.
} 
Alimenticios Canutos como mermeladas, choco canutos y frutas deshidratadas donde se siguen apalancando en el hecho de ser frutas tropicales exóticas que normalmente no se encuentran en C.A.B.A.

La estrategia se basará en el hecho de ser productos de frutas tropicales naturales, elaboradas con procesos artesanales únicos en el país de origen, en donde inicialmente se darán a conocer a través del mercado gastronómico natural en el que se aprovechara la masificación del consumidor final (comensales) como parte introductoria para luego dirigirse a otro tipo de canales como las tiendas naturistas y hoteles que valoren lo natural y quieran contar con un producto exclusivo.

\section{Posicionamiento}

Según los métodos de segmentación seleccionados; segmentación socio demográfico y segmentación por usuario no usuario ${ }^{21}$ se definió que el perfil del target son:

Hombres y mujeres entre 25 y 60 años ubicados en los CGP 1, 2, 14 Oeste y 14 Este pertenecientes al segmento C3, C2, A-B-C 1 de la Ciudad Autónoma de Buenos Aires los cuales buscan cuidar su salud con una alimentación sana y acuden regularmente a restaurantes de cocina natural.

Tienen un nivel de educación superior y regularmente salen a cenar con un amigo, pareja o con la familia. Sabe diferenciar los alimentos que más lo nutren y alguna vez ha sido asesorado por un nutricionista.

Es muy importante generar un vínculo en el consumidor donde relacione la marca con alimentación natural sana, para la cual se buscarán avales de entidades de nutrición como por ejemplo asociación argentina de nutricionistas y dietistas (AADYND), generando así una confianza en el consumidor final.

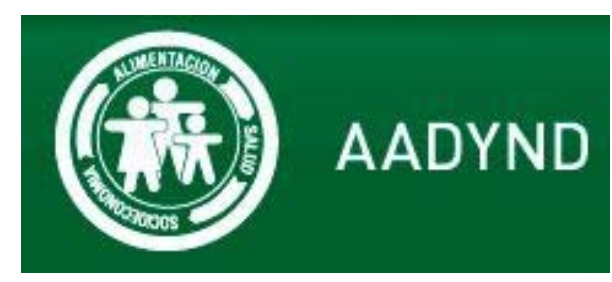

\footnotetext{
${ }^{21}$ Estrategias de segmentación y posicionamiento Dr. Gustavo E González - Universidad Nacional de la Plata
} 
Fuente: http://www.aadynd.org.ar/ información tomada el 5 de agosto de 2012.

También se piensan en otro tipo de estrategia, donde se contacten influenciadores de la marca como Nutricionistas o médicos reconocidos que apoyen la alimentación sana y den su testimonio después de probar el producto.

Hoy en día es muy común encontrar personas celiacas en la población argentina, es por eso que se decide incluir en los empaques el logo (sin gluten $100 \%$ garantizado) que distingue a los productos que están libres de gluten para que más personas estén seguras de probar un confite con sabores de frutas exóticas.

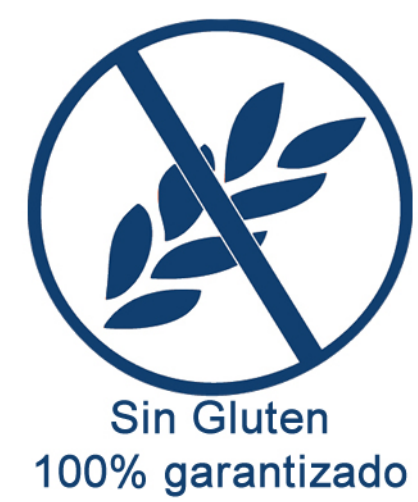

Al lograr incluir los dos logos mencionados anteriormente, se podrán reforzar en los consumidores el hecho de ser un producto $100 \%$ natural y que cuenta con una elaboración artesanal.

\section{m. Definición del Marketing Mix}

\section{Producto:}

Luego de haber realizado un trabajo de investigación de campo se puede afirmar que el producto lograría ser aceptado hablando en términos de composición, sabor y empaque*.

*En la investigación de campo que realicé - a manera de marketing de prueba conté con la colaboración de administradores o propietarios de restaurantes de comida natural en donde se dieron a probar los confites y proporcionaron la opinión del producto en una entrevista guiada.

Actualmente en el mercado colombiano se cuenta con cinco presentaciones del producto, en este proyecto se iniciara con el paquete de 200 unidades 
con dulces de 3 gramos y a mediano plazo introducir el paquete de 10 unidades de 5 gramos cada dulce.

El producto está hecho a base de leche, extractos de fruta, glucosa y azúcar, la duración del producto se estima en 4 y 6 meses a una temperatura ambiente conservando su sabor y textura. Es por eso que se decide transportar vía aérea el producto, ya que la prontitud en el envió es clave teniendo en cuenta su vigencia.

\section{Dulce de 3 gramos para Restaurantes *}

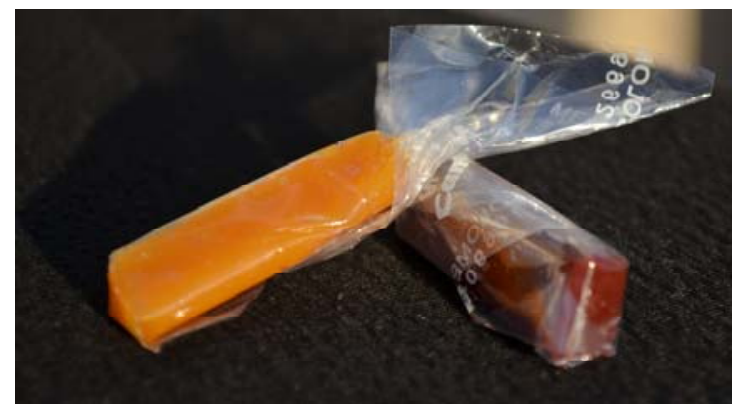

Paquete de 50 gramos Cliente Final 10 caramelos de 5 gramos cada uno.

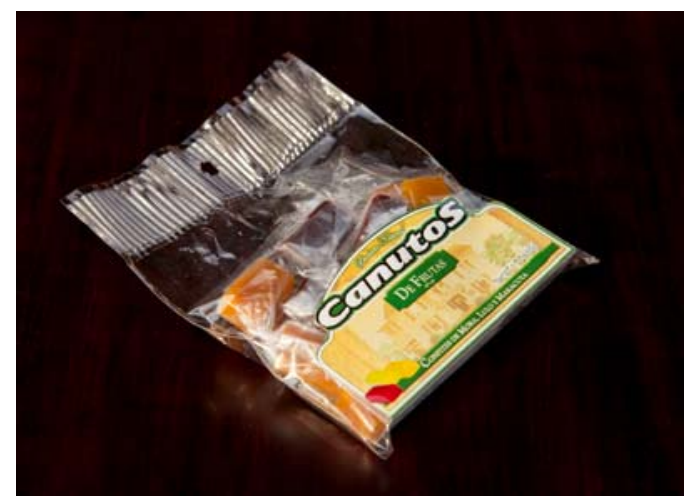

Paquete de 600 gramos Restaurantes 200 caramelos de 3,0 gramos cada uno.

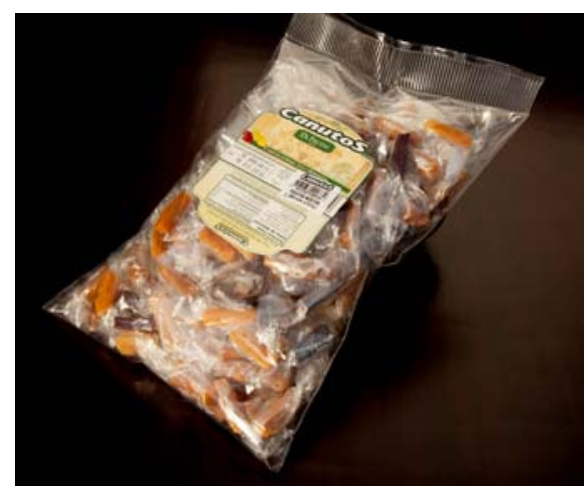




\section{Dispensador paquetes de $\mathbf{5 0}$ gramos (10 Und)}

para ubicar en restaurantes.

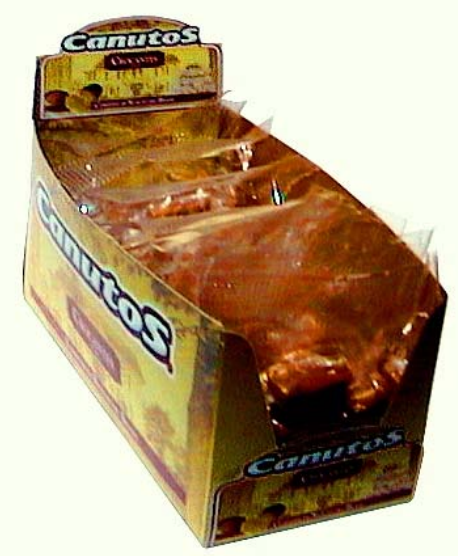

El producto cuenta además con una presentación para fechas especiales el cual se puede modificar a pedido del cliente. Este empaque puede ser de 50 o 75 gramos.
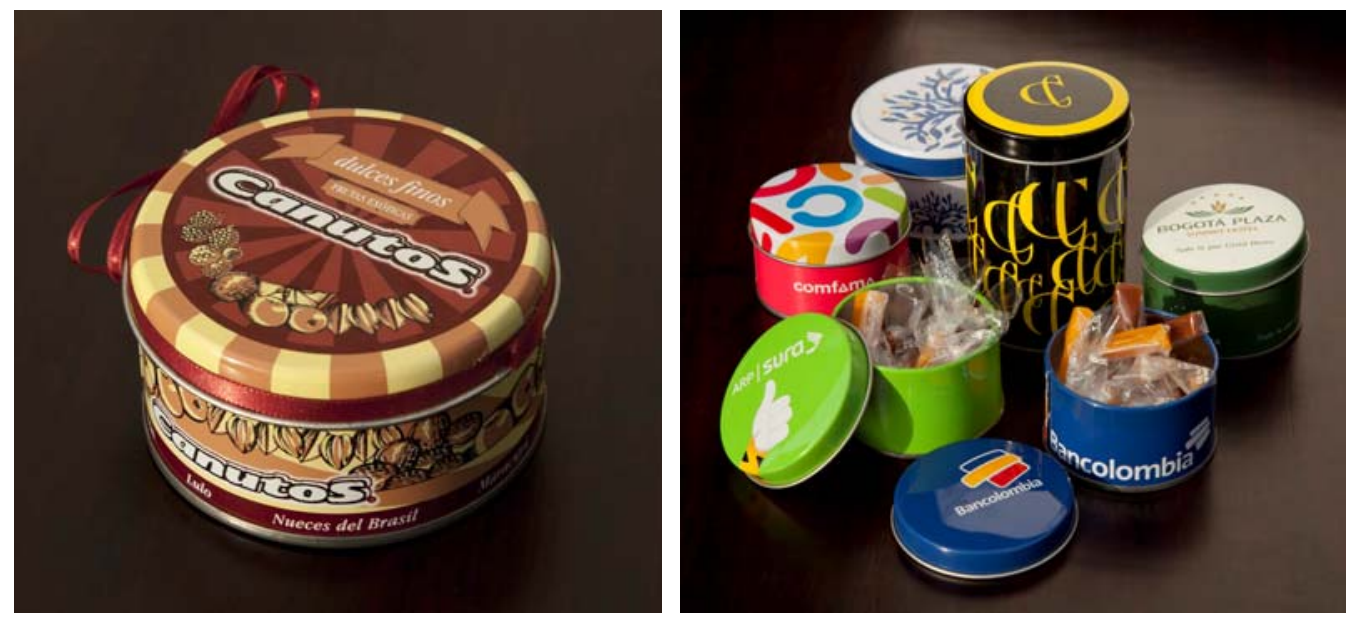

Fotografías: Samuel Restrepo

Se puede adaptar para la época de Pascua, para el día del amigo o para navidad como regalo corporativo.

El producto cuenta con una característica intangible clave la cual es, ser único en el mercado, por su variedad en sabores de frutas tropicales; lulo, uchuva, tamarindo, maracuyá entre otros, que no son comunes en los 
mercados locales de C.A.B.A generando un cierto interés en el consumidor final.

Luego de la investigación en campo se definió que es necesaria una adaptación en el empaque individual de celofán; este debe tener el nombre del sabor de la fruta impreso para buscar que los consumidores empiecen a tener un conocimiento de los diferentes sabores de las frutas tropicales y así facilite la apertura a otros productos con el mismo sabor como las mermeladas.

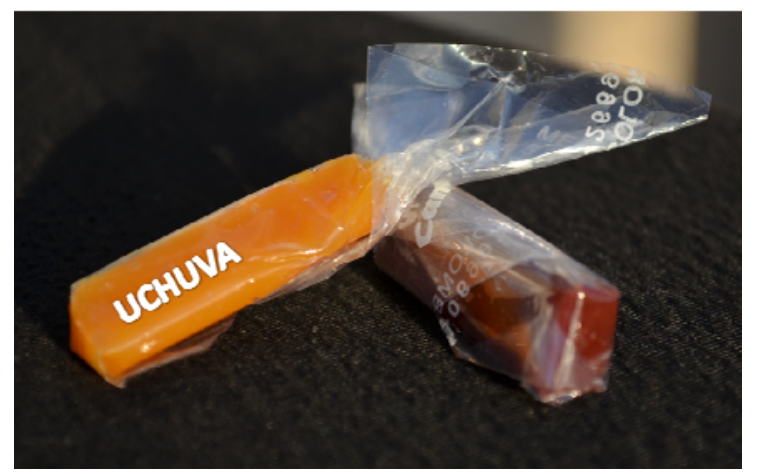

Fotografía Propia

Como lo menciona José Paris en su libro Marketing esencial, un enfoque latinoamericano, "la innovación es el arte de convertir las ideas y el conocimiento en producto"22 la estrategia de producto para el ciclo de vida será la innovación generando una extensión ${ }^{23}$ del producto en donde será clave el desarrollo de las relaciones con el cliente ya que gracias a él nace la extensión. (Ver anexo 3)

Es importante resaltar que cada empaque de 200 unidades contara con su etiqueta de información, en donde se podrá ver:

- Información Nutricional

- Código de Barras

- Fecha de vencimiento

- R.N.E - Registro Nacional de Establecimientos

- R.N.P.A - Registro Nacional de Productos y Alimentos

- Numero de lote.

- Producido por Productos Alimenticios Canutos

- Importado por Huerta Co S.R.L

\footnotetext{
22 Jose A Paris, Marketing Escencial un enfoque latinoamericano 2009 Edicion errepar.

${ }^{23}$ Warren Keegan , Marketing Global, Quinta edición
} 
El empaque debe contar con la estampilla de importación que al ser desprendida quedara inutilizada para cualquier otro producto.

El embalaje del producto se realizará en cajas de cartón corrugado de las siguientes medidas: 24 centímetros de alto x 40 centímetros de profundidad x 50 centímetros de ancho, con capacidad para 22 paquetes, peso neto de 16 kilogramos por caja y total de 480 kilogramos calculando 30 cajas por pallet de $120 \mathrm{~cm}$ largo x $100 \mathrm{~cm}$ ancho x $120 \mathrm{~cm}$ alto. (ANEXO 4)

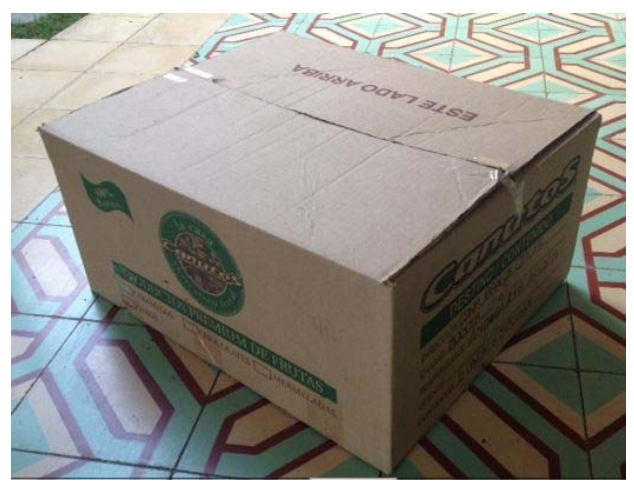

Foto: Samuel Restrepo, gerente de Productos Alimenticios Canutos

Se realizará un recubrimiento de seguridad con plástico (vinipel) y se etiquetará con código de barras como es debido para facilitar el proceso logístico exigido en la aduana.

\section{Precio:}

Al llegar a este punto, cabe anotar que en vista de la ausencia de la competencia directa y al no tener un valor referenciado, se estimó un valor promedio con base a la referencia el precio de venta del producto en Colombia para cálculos de flete y además como se puede ver en la tabla Nro 10 con la estructura de los costos directos y gastos de importación se logra llegar a un precio base para iniciar la penetración en el mercado. 
Tabla de simulación de costos de exportación No 10

\begin{tabular}{|c|c|}
\hline \multicolumn{2}{|c|}{ SIMULACION DE COSTOS DE EXPORTACIÓN } \\
\hline \multicolumn{2}{|c|}{ INFORMACION BASICA DEL PRODUCTO } \\
\hline Nombre Técnico o Comercial del producto & Confite de Fruta \\
\hline Posición arancelaria del país exportador & 1704.90 .90 .00 \\
\hline Posición arancelaria del país importador & 1704.90.20.499W \\
\hline Unidad comercial de Venta & Paquete de 200 unidades \\
\hline Valor unidad comercial & U\$S 30 \\
\hline Moneda & Dólares Americanos \\
\hline \multicolumn{2}{|c|}{ INFORMACION BASICA DEL EMBARQUE } \\
\hline País de Origen & Colombia \\
\hline Ciudad Punto de Embarque & Medellín - Antioquia \\
\hline País destino & Argentina \\
\hline Ciudad Punto de desembarque & Buenos Aires - Ezeiza \\
\hline No. De Unidades comerciales por embarque & 660 paquetes \\
\hline tipo de Empaque / Embalaje & Cajas de Cartón Corrugado \\
\hline Tipo unidad de Carga (Pallet) & $120 \times 100 \times 120$ centímetro \\
\hline \multicolumn{2}{|c|}{ DIMENSIONES } \\
\hline No. De Unidades por Pallet & 30 \\
\hline Largo Caja (mts) & 0,5 \\
\hline Ancho caja (mts) & 0,4 \\
\hline Alto caja (mts) & 0,24 \\
\hline Volumen total del Embarque ( m3) & 1,44 \\
\hline Peso por Caja (kilogramos) & 16 \\
\hline Peso total Pallet Cargado (Toneladas) & 0,48 \\
\hline \multicolumn{2}{|c|}{ BASE PARA LIQUIDAR FLETE (CARGA SUELTA) } \\
\hline Aereo & 0,24 \\
\hline Termino de Venta - Incoterm & FCA \\
\hline Forma de Pago & Letra de Cambio \\
\hline Plazo & 90 dias \\
\hline Tipo de Cambio Utilizado & 4,65 \\
\hline Porcentaje tasa de Seguro \% & 1 \\
\hline
\end{tabular}

* El Valor (FCA) Free carrier (Franco Transportista - libre transportista) será el INCOTERM acordado con el fabricante exportador donde entregará la mercadería en el aeropuerto de destino con los gastos pertinentes a la exportación cancelados. 


\begin{tabular}{|c|c|}
\hline $\begin{array}{l}\text { VALOR FCA Pallet de } 30 \text { cajas ( } 330 \text { Paquetes - } \\
66.000 \text { Unidades) Valor proporcionado por Team } \\
\text { Raiconet Logistic }\end{array}$ & US\$ 8.434 \\
\hline \multicolumn{2}{|c|}{ Tránsito Internacional } \\
\hline \multicolumn{2}{|l|}{ Costos Directos } \\
\hline Transporte Internacional (U\$S)/ Pallet de 15 cajas & US\$ 1.500 \\
\hline Valor CFR & US\$ 9.934 \\
\hline Seguro Internacional & US\$94 \\
\hline Valor CIP & US\$ 10.028 \\
\hline Manipuleo desembarque & US\$ 56 \\
\hline \multicolumn{2}{|l|}{ PAIS IMPORTADOR } \\
\hline \multicolumn{2}{|l|}{ Costos Directos } \\
\hline Transporte Local & US\$ 86 \\
\hline Almacenamiento x mes (1.44 m3) & US\$ 300 \\
\hline Seguro & US\$ 44 \\
\hline Valor DDU & US\$ 10.517 \\
\hline Documentación SIM & UD\$ 10 \\
\hline Aduaneros (Impuestos -Descritos Ref*) & US\$ 1.933 \\
\hline Agentes (Despacho de Aduana) & US\$ 150 \\
\hline Bancario & US\$ 150 \\
\hline Valor DDP Total & US\$ 12.760 \\
\hline
\end{tabular}

\begin{tabular}{|c|c|}
\hline \multicolumn{2}{|c|}{ CÁLCULO DE IMPUESTOS * } \\
\hline Valor CIP & US\$10.028 \\
\hline Derechos de Importación (20\%) & US\$2.000 \\
\hline Tasa estadística (0,5\%) & US\$0 \\
\hline Base Imponible IVA & US\$ 10.028 \\
\hline Total Impuestos & $\mathbf{\$ 2 . 0 0 0}$ \\
\hline
\end{tabular}

Teniendo en cuenta que la presente estructura está estimada para 30 cajas cada una con 22 paquetes de 200 unidades es decir 660 paquetes de Dulces canutos nacionalizada en Argentina cuesta, US $\$ 12.760$ aproximadamente.

Como lo sabemos el tema inflacionario en el escenario argentino no es muy favorable, por tal motivo se realizara una estandarización de precios trimestralmente calculando un porcentaje normal por mes de $1.4 \%$ en la 
inflación teniendo como referencia que para el mes de agosto del presente año fue de $1.64 \%^{24}$.

\section{Por tal motivo se presenta la tabla No 11 Matriz de precios y márgenes de ganancia}

\begin{tabular}{|c|c|c|c|c|c|c|}
\hline & $\begin{array}{c}\text { Margen de } \\
\text { Ganancia } \\
\text { por Canal }\end{array}$ & \begin{tabular}{|c|} 
Valor \\
Ganado por \\
cada \\
paquete \\
\end{tabular} & $\begin{array}{c}\text { Precio } 660 \\
\text { paquetes } \\
\text { de } 200 \text { Und. } \\
\text { (1 pallet de } \\
30 \text { cajas) } \\
\end{array}$ & \begin{tabular}{|c|} 
Precio \\
unitario \\
paquete de \\
200 \\
unidades \\
(US\$) \\
\end{tabular} & \begin{tabular}{|c|} 
Precio \\
unitario \\
paquete de \\
200 \\
unidades (\$ \\
Arg) \\
\end{tabular} & $\begin{array}{c}\text { Precio } \\
\text { unitario } \mathrm{x} \\
\text { confite } \\
\text { (US\$) }\end{array}$ \\
\hline Valor FCA Aeropuerto Colombia & & & US\$ 8.434 & US\$13 & $\$ 60$ & US\$ 0,065 \\
\hline Valor DDP Argentina Nacionalizado & & & US\$ 12.760 & US\$ 19 & $\$ 84$ & US\$0,096 \\
\hline $\begin{array}{c}\text { Precio de venta cliente Directo } \\
\text { (Restaurantes) }\end{array}$ & $35 \%$ & US\$ 7.5 & US\$ 17.226 & US\$ 26,50 & $\$ 123$ & US\$ 0,132 \\
\hline Precio de venta a representantes. & $25 \%$ & US\$ 5.5 & US\$14.873 & UD\$ 24.50 & $\$ 113$ & US\$ 0,122 \\
\hline
\end{tabular}

\section{Fuente: Cálculos Propios}

Teniendo en cuenta la información presentada, se puede definir que el precio para un paquete de 200 unidades es de $\$ 123$ pesos para venta cliente directo (restaurantes) y de $\$ 113$ pesos para representantes de ventas tema que se mencionara en la variable Plaza del Mix de Marketing.

La estimación de este precio (\$123 pesos), para una penetración en el mercado, se ajusta a la estrategia general, al establecer una relación comercial con cada cliente ${ }^{25}$ donde se busca acercarse cada vez más al consumidor final (los comensales).

\section{Plaza}

Como se sabe el proyecto inicia con una cobertura en C.A.B.A y los clientes estarán ubicados en 4 CGP identificados en el capitulo anterior; 1 , 2 Norte, 14 Oeste y 14 Este. Aprovechando este punto y luego de contar con la mercadería en la bodega de alimentos, el encargado de marketing y ventas realizará los recorridos de entrega para poder empezar a tener un contacto directo según la estrategia y poder tener una retroalimentación activa donde el negocio se pueda adaptar a las necesidades de los clientes.

Se plantea la idea que a los 6 meses de operaciones se pueda generar otro canal de distribución, con representantes de ventas los cuales se otorga un

\footnotetext{
${ }^{24}$ http://www.ambito.com/diario/noticia.asp?id=653555 Información tomada el 14 de septiembre de 2012.

${ }^{25}$ Licenciado Aldo F. Albarellos, Marketing y Competitividad, Editorial Pearson, Buenos Aires 2009
} 
descuento de $10 \%$ para ofrecer el producto en Hoteles, tiendas naturistas y otros mercados.

El precio del producto para estos representantes de ventas será del \$113 + IVA dándoles un $10 \%$ de descuento sobre el precio establecido para cliente final \$123 + IVA (restaurantes). Con este plan de precios se tiene planeado lograr una cobertura más amplia en C.A.B.A y a mediano plazo generar puntos de venta.

Paso a Paso:

- Se tomaran los pedidos de compra de cada cliente, personalmente o a través de los diferentes medios; teléfono, e-mail, página web, allí se debe tener clara la fecha de entrega y plazo de pago.

- Relevamiento de orden de compra al fabricante de Dulces Canutos

- Envió de la mercadería

- Recepción y almacenamiento en bodega fría

- Distribución por parte del aliado estratégico.

\section{Promoción}

Como se sabe la marca está influenciada por una cultura natural en donde se resalta la elaboración del producto en forma artesanal y con ingredientes $100 \%$ naturales, dado este hecho se aplicará un estilo gráfico de naturaleza y donde los colores vivos de las frutas, generarán impacto en la identidad corporativa, resaltando la variedad de las frutas tropicales que ofrece el producto.

La identidad corporativa de la página web también tendrá implícito estilo gráfico definido, en donde se verán imágenes de la casa Canutos, sus trabajadores y los productos en sus diferentes empaques.

La pagina web tendrá un espacio exclusivo en donde se muestren los restaurantes donde estarán presentes los dulces, siendo este un beneficio más obtenido por los clientes.

La generación de los links de las redes sociales como Facebook, Twitter y YouTube en la página web, es un punto clave para el surgimiento de una estrategia de comunidad en donde se sigue buscando el contacto directo con el cliente final. 
Ver anexo No 5 con el diseño de primer pantallazo.

La realización de un brochure o folleto es indispensable para la labores de venta directa, este material promocional tendrá una código $\mathrm{QR}{ }^{26}$ el cual direccionará de inmediato a la página web www.canutosnaturales.com en donde se tendrá la opción de vincularse en las redes sociales .

Ver anexo No 6 Diseño sugerido de Brochure.

La idea de los códigos QR se plantea, por que el target se adapta a este tipo de recursos tecnológicos ya que por lo general los clientes que visitan estos restaurantes, portan Smartphone y tienen como realizar la lectura del código QR.

Pocos serán los recursos en promoción masiva, así que se opta por realizar degustaciones esporádicas una vez al mes, en los mercados orgánicos como por ejemplo; Sabe la tierra en la estación de San Fernando en el tren de la costa, Mercado Punto Verde, El Galpón y Mercado Bonpland a donde llega gente que está interesada por alimentarse saludablemente y está dentro del target del producto.

Se debe realizar la gestión para contar con el permiso de realizar degustaciones en cada mercado. Se propondrá gestionar un canje con cada representante del mercado proponiéndole la pauta de la página Web de cada uno de forma gratuita durante determinado tiempo por una presencia mensual de la representante de ventas realizando degustación.

Esta degustación será realizada por una representante de ventas quien contara con el Banner de la marca, ver anexo No 7, además la supervisión del encargado de marketing y ventas será clave para la generación de base de datos a través de medios electrónicos

Las tarjetas de presentación, carpetas y papel membrete estará diseñado bajo el mismo estilo grafico ver anexo No 8 con el modelo propuesto de tarjeta de presentación.

A continuación veremos un estimado de costos para la promoción del Producto proyectado a 5 años, haciendo referencia al contrato de representación comercial del producto firmado al inicio del proyecto.

\footnotetext{
${ }^{26}$ http://www.codigos-qr.com/ información tomada el 19 de septiembre de 2012.
} 


\section{Cotización de precios sin IVA para la Promoción del Producto Tabla No 12}

\begin{tabular}{|c|c|c|c|c|}
\hline Tarjetas de presentación & Unidades & $\begin{array}{l}\text { Precio Unitario en } \\
\text { Pesos Argentinos }\end{array}$ & $\begin{array}{l}\text { Valor total } \\
\text { Pesos Arg }\end{array}$ & $\begin{array}{l}\text { Precio total } \\
\text { en Dólares }\end{array}$ \\
\hline Producción e impresión & 1000 & 0,465 & 465 & US\$ 100 \\
\hline Diseño y Arte & 1 & 100 & 100 & US\$ 21 \\
\hline http://www.imprimime. & aid & \multicolumn{2}{|c|}{ Total Tarjetas de presentación } & US\$ 121 \\
\hline
\end{tabular}

\begin{tabular}{|c|c|c|c|c|}
\hline Brochure & Unidades & $\begin{array}{c}\text { Precio } \\
\text { Unitario en } \\
\text { Pesos } \\
\text { Argentinos }\end{array}$ & $\begin{array}{l}\text { Valor total } \\
\text { Pesos Arg }\end{array}$ & $\begin{array}{l}\text { Precio total } \\
\text { en Dólares }\end{array}$ \\
\hline $\begin{array}{l}\text { Producción e } \\
\text { impresión }\end{array}$ & 1000 & 1,17 & 1170 & US\$ 250 \\
\hline Diseño y Arte & 1 & 350 & 350 & US\$ 75 \\
\hline http://www.imprim & m.ar/ & \multicolumn{2}{|c|}{ Total Brochure } & US\$ 325 \\
\hline
\end{tabular}

\begin{tabular}{|c|c|c|c|c|}
\hline Pagina Web (incluye) & Unidades & $\begin{array}{c}\text { Precio } \\
\text { Unitario en } \\
\text { Pesos } \\
\text { Argentinos }\end{array}$ & $\begin{array}{l}\text { Valor total } \\
\text { Pesos Arg }\end{array}$ & $\begin{array}{l}\text { Precio total } \\
\text { en Dólares }\end{array}$ \\
\hline Diseño & \multirow{4}{*}{1} & \multirow{4}{*}{1900} & \multirow{4}{*}{1900} & \multirow{4}{*}{ US\$400 } \\
\hline Posicionamiento & & & & \\
\hline 1 año de Hosting & & & & \\
\hline Estrategia Adwors & & & & \\
\hline http://sitioswebinternet & m.ar/ & \multicolumn{2}{|c|}{ Total Pagina Web } & US\$400 \\
\hline
\end{tabular}

\begin{tabular}{|c|c|c|c|c|}
\hline $\begin{array}{l}\text { Banner Corporativo } \\
\text { (160 x } 60 \text { centímetros) }\end{array}$ & Unidades & $\begin{array}{c}\text { Precio } \\
\text { Unitario en } \\
\text { Pesos } \\
\text { Argentinos }\end{array}$ & $\begin{array}{l}\text { Valor total } \\
\text { Pesos Arg }\end{array}$ & $\begin{array}{l}\text { Precio total } \\
\text { en Dólares }\end{array}$ \\
\hline Producción e impresión & 1 & 170 & 170 & US\$ 37 \\
\hline Diseño y Arte & 1 & 350 & 350 & US\$ 75 \\
\hline http://www.imprimime.c & n.ar/ & \multicolumn{2}{|c|}{ Total Brochure } & US\$ 112 \\
\hline
\end{tabular}

\begin{tabular}{|l|r|}
\hline \multicolumn{2}{|c|}{ Resumen de Costos Promocionales en Dólares } \\
\hline Tarjetas de Presentación & US\$121 \\
\hline
\end{tabular}




\begin{tabular}{|l|c|}
\cline { 2 - 2 } Brochure & US\$325 \\
\hline Pagina Web & US\$400 \\
\hline Banner Corporativo & US\$112 \\
\hline Total & US\$958 \\
\hline
\end{tabular}

\begin{tabular}{|c|c|c|c|c|c|}
\hline \multirow[b]{2}{*}{ Tarjetas de presentación } & \multicolumn{5}{|c|}{ Precio total en Dólares } \\
\hline & Año 1 & Año 2 & Año 3 & Año 4 & Año 5 \\
\hline Producción e impresión & $\$ 100$ & $\$ 100$ & $\$ 85$ & $\$ 85$ & $\$ 85$ \\
\hline Diseño y Arte & $\$ 25$ & & $\$ 30$ & & \\
\hline \multicolumn{6}{|l|}{ Bruchure } \\
\hline Producción e impresión & $\$ 250$ & $\$ 250$ & $\$ 200$ & $\$ 200$ & $\$ 200$ \\
\hline Diseño y Arte & $\$ 75$ & & $\$ 60$ & & \\
\hline \multicolumn{6}{|l|}{ Pagina Web (incluye) } \\
\hline Diseño & \multirow{3}{*}{$\$ 400$} & $\$ 45$ & $\$ 40$ & $\$ 40$ & $\$ 40$ \\
\hline Posicionamiento & & $\$ 25$ & $\$ 27$ & $\$ 30$ & $\$ 35$ \\
\hline 1 año de Hosting & & $\$ 77$ & $\$ 84$ & $\$ 93$ & $\$ 102$ \\
\hline \multicolumn{6}{|l|}{$\begin{array}{l}\text { Banner Corporativo } \\
\text { ( } 160 \times 60 \text { centímetros) }\end{array}$} \\
\hline Producción e impresión & $\$ 35$ & $\$ 35$ & $\$ 28$ & $\$ 28$ & $\$ 28$ \\
\hline Diseño y Arte & $\$ 75$ & & $\$ 60$ & & \\
\hline Total X Año USD\$ & $\$ 960$ & $\$ 430$ & $\$ 503$ & $\$ 353$ & $\$ 353$ \\
\hline Total Pesos Argentinos & $\$ 4.464$ & $\$ 2.000$ & $\$ 2.339$ & $\$ 1.641$ & $\$ 1.641$ \\
\hline
\end{tabular}

Fuente: Cálculos Propios

Se define que para la promoción inicial del proyecto se requiere una inversión de US\$4.464. Y para los años siguientes un presupuesto de mantenimiento y control.

Estos valores estimados para la promoción del producto serán tenidos en cuenta en el capitulo siguiente en el cash flow proyectado. 


\section{IV.Conclusiones}

\section{n. Presupuesto y control}

Tomando la información dada en el presenta trabajo se desglosan los ítems que hacen parte de la inversión inicial del Plan de Marketing Internacional para la Exportación de dulces canutos de origen Colombiano a Ciudad Autónoma de Buenos Aires - Capital Federal - de la República Argentina.

Presupuesto Inicial del Proyecto Tabla No 13

\begin{tabular}{|c|c|c|c|}
\hline Item & Qué & Donde & $\begin{array}{c}\text { Costos istimado } \\
\text { (\$ARG) }\end{array}$ \\
\hline $\begin{array}{l}\text { Selección y } \\
\text { Contratacion de } \\
\text { personal }\end{array}$ & $\begin{array}{c}\text { Avisos en redes de busqueda } \\
\text { de empleo Zona Jobs y } \\
\text { Bumerang) }\end{array}$ & C.A.B.A. & $\$ 8.800$ \\
\hline \multirow{6}{*}{$\begin{array}{c}\text { Inscripciones Registros } \\
\text { y Licencias }\end{array}$} & Inscripcion como comerciante & $\begin{array}{l}\text { Registro publico de } \\
\text { comercio }\end{array}$ & $\$ 500$ \\
\hline & $\begin{array}{c}\text { Inscripcion Direccion General } \\
\text { Impositiva }\end{array}$ & $\begin{array}{c}\text { Administracion } \\
\text { Federal de Ingresos } \\
\text { Publicos } \\
\end{array}$ & $\$ 0$ \\
\hline & $\begin{array}{c}\text { Inscripcion Registro de } \\
\text { Importadores y Exportadores*. }\end{array}$ & $\begin{array}{c}\text { Direcion General de } \\
\text { Aduanas }\end{array}$ & $\$ 0$ \\
\hline & $\begin{array}{l}\text { Inscripcion en el resgitro de } \\
\text { importadores del SENASA }\end{array}$ & SENASA & $\$ 0$ \\
\hline & $\begin{array}{c}\text { Resgistro de Producto } \\
\text { Alimenticio* }\end{array}$ & INAL & $\$ 300$ \\
\hline & Registro de Marca & $\mathrm{INPI}$ & $\$ 1.000$ \\
\hline Infraestructura & $\begin{array}{c}\text { Alquiler y gastos de oficina } \\
\text { comercial }\end{array}$ & C.A.B.A. & $\$ 9.000$ \\
\hline Infraestructura & $\begin{array}{c}\text { Muebles y Equipos de oficina - } \\
\text { Computadores }\end{array}$ & C.A.B.A. & $\$ 5.000$ \\
\hline $\begin{array}{l}\text { Capacitacion y } \\
\text { Entrenamiento }\end{array}$ & $\begin{array}{c}\text { Asesoria de contador y } \\
\text { abogado para constitucion } \\
\text { empresarial }\end{array}$ & C.A.B.A. & $\$ 1.700$ \\
\hline Mercaderia & $\begin{array}{l}\text { Primer envio de mercaderia } \\
\text { (pallet con } 30 \text { cajas) }\end{array}$ & C.A.B.A. & $\$ 39.215$ \\
\hline Publicidad y Pomocion & $\begin{array}{l}\text { Pagina de Internet y material } \\
\text { promocional }\end{array}$ & C.A.B.A. & $\$ 20.675$ \\
\hline \multicolumn{3}{|c|}{ VCIAL EN PESOS ARC } & $\$ 86.190$ \\
\hline
\end{tabular}

Fuente: Autor.

Luego de detallar el presupuesto inicial del proyecto podemos concluir que la inversión es de $\$ 86.190$ pesos argentinos, teniendo en cuenta que para la ejecución inicial se pueden presentar varios elementos imprevistos que eleven un poco el valor. 
En el anexo No 10 se podrá ver el flujo de caja detallado con una proyección a 5 años para buscar el punto de equilibrio buscado.

\section{Proyecciones (ventas y costos)}

Para realizar las proyecciones de ventas (anexo No 9) se tuvo en cuenta la tabla No 5 de proyección de la demanda a 5 años y la tabla No 11. Matriz de precios y ganancias, para los costos el precio DDP (Delivered Duty Paid) del valor de un paquete de 200 unidades nacionalizado en Argentina donde se estima en USD\$19, lo que permite realizar las proyecciones.

A futuro con la compra de mayor volumen de producto se puede negociar un descuento con el fabricante colombiano, vale resaltar que se pretende importar otros productos como mermeladas y frutas deshidratadas al iniciar el tercer año de trabajo.

\section{o. Análisis Financiero}

En este análisis (anexo No 9) se asienta en el análisis de flujo de caja proyectado a 5 años implicando los ingresos y egresos del anexo No 8 así como también el valor de inversión inicial y el aporte de los socios para la concepción del plan de marketing internacional.

Para este análisis solo se tuvo en cuenta la venta de Confites en paquetes de 200 unidades en los 5 años, es importante resaltar que el contrato de representación es de 3 años pero con posibilidad de renovación lo cual dará vía a la importación de otros productos como mermeladas, chocho canutos y frutas deshidratadas, haciendo más grande el catálogo de productos para generar más ventas.

En el siguiente punto se podrá ver datos relevantes del plan que permitirán declarar la viabilidad financiera del proyecto.

\section{p. Indicadores Financieros VAN - TIR}

Valor Actual Neto VAN: este indicador mide los flujos de los futuros ingresos y egresos que tendrá el proyecto, para determinar si luego de descontar la inversión inicial, quedan ganancias. Si el indicador es positivo el proyecto es viable. 
La fórmula que se aplica para calcular este indicador es:

$$
\mathrm{VAN}=\sum_{t=1}^{n} \frac{V_{t}}{(1+k)^{t}}-I_{0}
$$

Donde,

$V_{t} \quad$ Flujo de caja de cada periodo

$I_{0} \quad$ Valor de la Inversión

$n \quad$ Numero de periodos considerado

$k \quad$ Tipo de interés

Teniendo en cuenta el concepto ilustrado, el VAN del proyecto según los flujos de caja y el monto de inversión representados en el anexo 8 da como resultado el valor positivo USD\$47.926. Usando una tasa de renta fija del $\% 5$ al a cual se le descuentan los flujos futuros, el indicador nos muestra que el proyecto bajo las condiciones pautadas genera beneficios, logrando recuperar la inversión inicial.

\section{Tasa Interna de Rentabilidad - TIR}

Se define como la tasa de rentabilidad esperada por medio de la cual se recupera la inversión del proyecto.

Si se obtiene un TIR mayor así será la rentabilidad del negocio, esta deberá ser mayor al porcentaje a una tasa mínima o tasa de corte denominada así cuando existe inversión sin riesgo, esta la tasa de rentabilidad mínima a la cual los inversores del proyecto destinaria el capital para su ejecución.

Realizando la operación teniendo en cuenta el flujo de caja proyectado (anexo 10) y la inversión realizada se obtiene un resultado positivo del $28 \%$ logrando un rendimiento aceptable que complementado con el resultado del VAN toleran una viabilidad financiera para el plan de marketing internacional de Dulces Canutos bajo los condiciones expuestas con la venta de solo un producto.

\section{q. Punto de equilibrio}

Teniendo en cuenta la importancia de este tipo de proyectos es necesario calcular el punto de equilibrio, el cual nos muestra el volumen mínimo de 
ventas que debe tener la empresa, para que sea sustentable con la estructura propuesta en el proyecto.

Tomando datos de flujos de fondos (anexo 8), donde se determinan como costos fijos (CF) tales como recursos humanos, gastos administrativos, de comercialización y financieros, precio de venta por unidad (PV) y costo variable por unidad (CVU) (la diferencia de estos dos últimos llamado contribución marginal por unidad vendida) representado en la siguiente fórmula:

$$
P E Q \text { unidades })=\frac{\text { Costos } F \text { yos }(C F)}{P V-C V U}
$$

Resultados de la operación Punto de Equilibrio:

El punto de equilibrio dio como resultado en paquetes de 200 unidades, 14.983 paquetes, que tendrían que vender como mínimo para empezar a tener beneficios. Esto es calculado a 5 años de operaciones con un precio de venta de USD $\$ 26,50$

Este punto de equilibrio representa el $87 \%$ del total estimado a importar en los cinco años proyectados teniendo como meta 17.143 paquetes de 200 unidades, lo cual es un margen aceptable para las cantidades que se pretenden comercializar bajo los supuestos mencionados. 


\section{CONCLUSIONES GENERALES}

El aumento en el número de adeptos a la nueva cultura de alimentación sana, el constante crecimiento del sector gastronómico en Ciudad Autónoma de Buenos Aires y la falta de variedad de frutas que se ven en los mercados locales fueron el eje central del proyecto, enmarcando el análisis del consumidor y sus hábitos de consumo, al igual que la ubicación de los sectores en donde se están generando más restaurantes de comida sana.

Los diferentes análisis, tanto del mercado de origen (Colombia) como el mercado meta (C.A.B.A), los factores críticos y los datos más relevantes, permitieron realizar una estrategia de precio adecuada para la incursión en el negocio, adaptándose a los canales de distribución contemplados en el PEMI.

Es así como después de un proceso de análisis y recopilación de información se llaga a las conclusiones que se detallarán a continuación:

$>$ Para la estructura organizacional de la empresa se plantea un contrato de representación comercial internacional con el fabricante colombiano Productos Alimenticios Canutos, en donde se recibe un permiso de 3 años inicialmente para la representación y comercialización de Dulces Canutos conformando un equipo de 3 personas; administración y contabilidad, marketing y ventas, logística y distribución, quienes además contarán con la asesoría externa para conformación de la sociedad y la gestión impositiva.

> La estructura financiera del proyecto será conformada por capital propio en dos cuotas; primer y segundo año de operación.

$>$ El ambiente actual para la importación de artículos de confitería a la Republica Argentina y su comercialización en C.A.B.A está en buenas condiciones, mostrando agilidad en los procesos y afirmando que la cooperación beneficiara los oferentes como los demandantes.

> La economía argentina ha mostrado varias fortalezas en los últimos años gracias a la capacidad de producción instalada dándole un dinamismo al sector alimenticio.

La exportación de confituras provenientes de Colombia y con destino en La Republica Argentina ha crecido un 18\% de 2006 al 2011, dando confianza al proyecto y generando un incremento de comercio bilateral entre los dos países. 
$>$ El proyecto cuenta con una ventaja competitiva gracias a un enfoque de nicho, siendo este el notable crecimiento de la cultura de alimentación sana en Ciudad Autónoma de Buenos Aires, generando una demanda incipiente en el mercado gastronómico natural de C.A.B.A.

$>\quad$ La variedad de frutas exóticas como el lulo, tamarindo, uchuva y maracuyá despiertan curiosidad en los clientes dándole un cierto atractivo al producto, permitiéndole a los clientes conocer otros sabores de Latinoamérica y dando apertura a la importación de más productos como; mermeladas, choco canutos y frutas deshidratadas.

$>$ El producto inicial será confites de fruta natural Canutos presentado en paquetes de 200 unidades con un surtido mixto de sabores (lulo, maracuyá, tamarindo, uchuva, mora, limón, arequipe y nuez) y con un peso de 3 gramos, también el paquete de 10 unidades mixtas de 5 gramos cada una.

> Los objetivos del plan de marketing siempre están enfocados al crecimiento de la ventas para lograr una diversificación de productos de frutas exóticas naturales donde se resalta la elaboración artesanal y el aval de la AANDYN Asociación Argentina de Dietistas y Nutricionistas para la generación de seguridad y confianza en los consumidores finales.

$>$ Se conoce que el producto es libre de gluten, por esto se pondrá el logo "libre de gluten 100 \% garantizado"

$>$ Se realizó una segmentación inicial de mercado meta en donde se definieron 4 centros de gestión y participación como mercado de apertura (CGP 1, 2, 14 Oeste y 14 Este).

> Se define que el target del proyecto son; hombres y mujeres entre 25 y 60 años pertenecientes al segmento C3, C2, A-B-C 1 de la Ciudad Autónoma de Buenos Aires los cuales buscan cuidar su salud con una alimentación sana. Tienen un nivel de educación superior y regularmente salen a cenar con un amigo, pareja o con la familia. Sabe diferenciar los alimentos que más lo nutren y alguna vez ha sido asesorado por un nutricionista.

$>$ El mercado alterno serán los hoteles, entidades corporativas y tiendas de nutrición de las zonas previamente mencionadas del cual se encargaran los representantes de ventas.

La cuantificación de la demanda está validada sobre 50 restaurantes que se registran activos según fuentes secundarias, la meta inicial es 
de 20 restaurantes para iniciar el año y como cierre de año finalizar con presencia en 35 restaurantes logrando un $70 \%$ de la demanda inicial.

- Con relación al plan de Marketing Mix del producto se establecieron los siguientes puntos:

$>$ El producto inicial serán confites de fruta natural envueltos en celofán, en paquetes de 200 unidades multi sabores (lulo, maracuyá, tamarindo, uchuva, mora, limón, arequípe y nuez), empacados en cajas de cartón corrugado por 22 unidades.

$>$ El precio final definido es de US\$26,50 Dólares que corresponden a $\$ 123$ pesos con un margen de ganancia de 35\% a la Importadora y representante comercial Huerta Co S.R.L

$>$ Se venderá directamente a los restaurantes de cocina natural y a través de representantes de ventas en mercados alternos como; Hoteles, entidades corporativas y tiendas naturistas brindándoles un descuento del $10 \%$ sobre el precio a consumidor final.

$>$ Se realizará una promoción a través degustaciones exclusivas de productos, en los mercados orgánicos y naturales con las representantes de ventas, además la ejecución de la página web y los recursos digitales como los códigos QR que apoyarán la labor promocional del producto.

$>$ Teniendo en cuenta que la proyección del proyecto se hizo pensando en la venta de un solo producto, arrojó como resultado un indicador TIR del $17 \%$ y un VAN del USD\$ 31.697 lo que genera una viabilidad financiera para ejecutar el Plan de Marketing Internacional.

- La cantidad mínima de venta durante el proyecto se estima en 14.983 paquetes de 200 unidades lo cual representa 681 cajas de 22 paquetes.

$>$ El capital correspondiente a la inversión inicial tendrá un periodo de recuperación de 4 años, 8 meses y 26 días teniendo en cuenta que solo se proyecta la venta de un producto.

Considerando todos los puntos estudiados se concluye que el negocio de importación y comercialización de Confites Canutos provenientes de Medellín Colombia a Ciudad Autónoma de Buenos Aires de la Republica Argentina es un negocio con varios puntos a favor para el éxito comercial. 


\section{BIBLIOGRAFIA}

- Aldo F. Albarellos, Marketing y Competitividad, Editorial Pearson, Buenos Aires 2009

- Mag. Ing. José A. París - Marketing Esencial Un enfoque latinoamericano - Buenos Aires 2009 - editorial errepar.

- Néstor Aleksink y Gustavo Segré - Fundamentos para un proyecto de exportación - Segunda Edición

- Informe sector alimentos industrializados - Golosinas y Productos de Confitería 2011 - Dirección de Oferta Exportable - Dirección General de Estrategias de Comercio Exterior Subsecretaría de Desarrollo de Inversiones y Promoción Comercial. Ministerio de Relaciones Exteriores y Culto.

- Programa de apoyo a la reconversión empresarial para las exportaciones módulo mejora de la competitividad sectorial sector alimentos gourmet Diagnóstico del Sector. ALPHA Estudio de Economía y Negocios S.A. (Agosto de 2006).

- Aladi - Mercosur, La Intergracion Regional, Hector Froiz Domingo Mazza, Gerardo Woscoboinik

- Programa de Vacancia (PAV 103)- Trabajo, desarrollo y diversidad Una investigación sobre metodologías y políticas de desarrollo local con acento en la generación de empleo / ingresos - Documento final sobre La Ciudad Autónoma de Buenos Aires Cecilia Del Bono ceciliadb@uolsinectis.com.ar

- Warren Keegan, Marketing Global, Quinta Edición Prents Hall, Madrid 1997.

- Otros Documentos:

- Dr. Gustavo E. González. Estandarización Nivel Socioeconómico. Presentación expuesta en el curso Información para decisiones de Marketing - Facultad de Ciencias Económicas UNLP 2011.

- Dr. Cristina Zapata - Presentación - Marketing Internacional en mercados Internacionales - Universidad Nacional de La Plata 2012. 


\section{DOCUMENTOS DE INTERNET}

- Rrestaurantes de cocina natural 2012 en www.guiaoleo.com.ar, www.restorando.com, www.viaresto.clarin.com .

- Mapa Económico Ciudad Autónoma de Buenos Aires 2012 http://mapoteca.educ.ar

- Estructura Social de La Argentina - Definición del Índice NES - IMAR 2002 en http://www.eclac.cl/publicaciones/xml/7/11307/L1772e-P.pdf

- AIERA (asociación de importadores y exportadores de la republica argentina) 2012 en www.aiera.org.ar

- Proexport Colombia, Promoción de Turismo Inversión y Exportación 2012 en http://www.proexport.com.co

- Guía para exportar productos desde Colombia 2012 en http://www.colombiatrade.com.co

- Tabla de Exportaciones totales colombianas por partida arancelaria 1704. 2011 en http://www.dane.gov.co.

- Asociación de Bancos en Argentina 2012 en; www.aba-argentina.com

- Secretaria General de la comunidad andina, documentos de información, 2004 en, http://intranet.comunidadandina.org/Documentos/DInformativos/SGdi671 .pdf

- Logo de la Asociación Argentina de dietistas y nutricionistas 2012 en http://www.aadynd.org.ar/

- Logo Marca País Colombia Co 2012 en http://www.colombia.co .

- Fotografía de referencia para ciclo de producto 2012 en http://blogs.lanacion.com.ar/cocina-amateur/carne-de-pescado/salmonrosado-sobre-salsa-de-frutos-tropicales-a-la-manera-de-diego-bianchi/

- Indicadores de Inflación argentina para cálculos propios 2012 en, http://www.ambito.com/diario/noticia.asp?id=653555

- Información sobre la Estructura Logística Argentina Banco Mundial 2012 en 
http://lpisurvey.worldbank.org/international/scorecard/radar/254/C/ARG/2 012/C/ARG/2010/C/ARG/2007?sort=asc\&order=Customs\#datatable

- Información sobre Códigos QR, estrategia de promoción 2012 en; http://www.codigos-qr.com/

- Medios de Pago vía Web en Argentina 2012 en https://www.paypal.com/ar/cgi-bin/webscr?cmd= wp-standard-overviewoutside

- Cotizaciones de materiales promocionales y pagina web 2012 en www.sitioswebinternet.com.ar y www.imprime.com.ar. 


\section{ANEXO No 1}

\section{MAPA ECONÓMICO DE C.A.B.A}

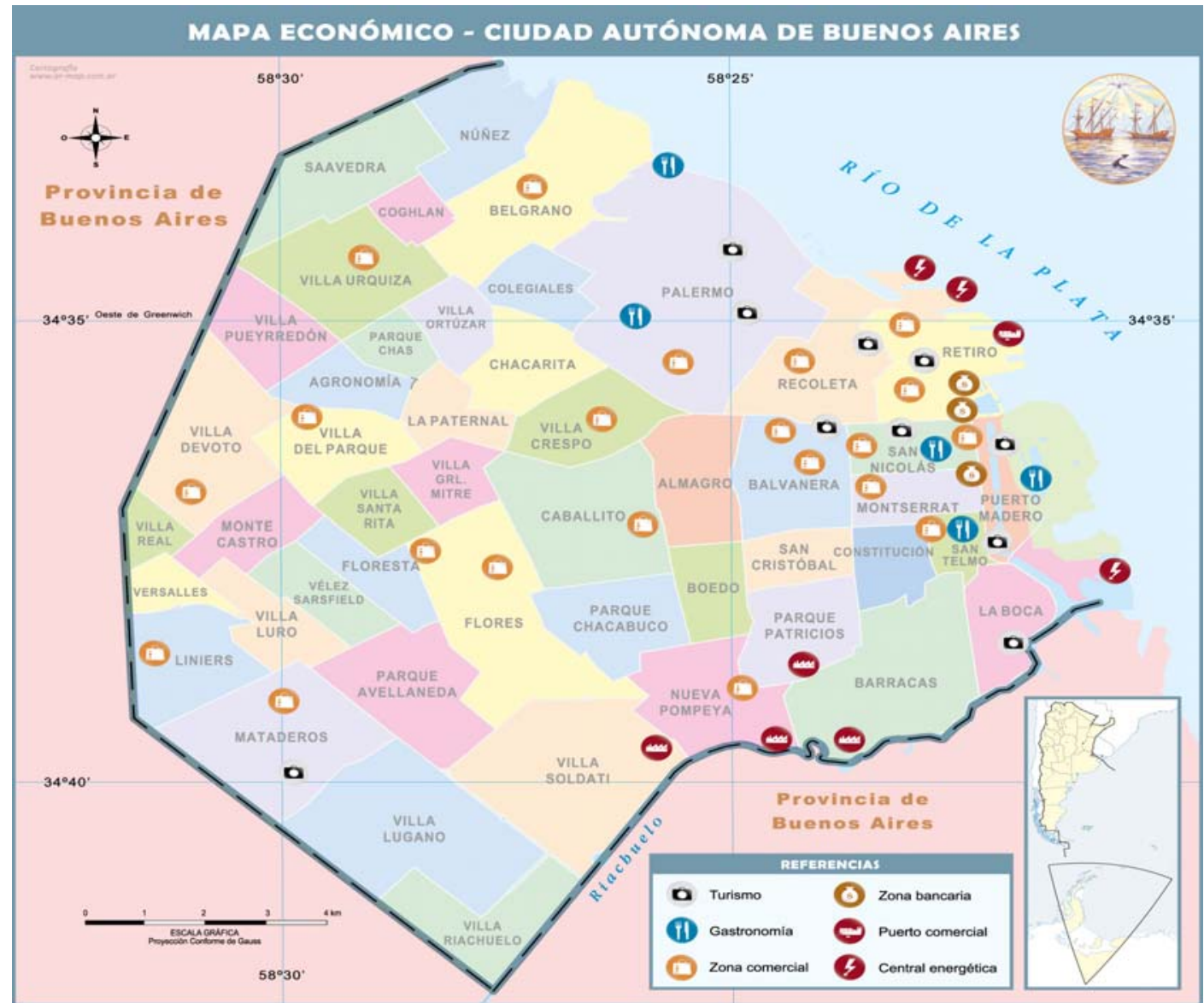

Fuente: http://mapoteca.educ.ar/ información tomada el 28 de agosto de 2012. 


\section{ANEXO 2 \\ CONVENI O DE COMERCI ALIZACI ON Y DI STRI BUCI ÓN \\ I NTERNACI ONAL}

Entre

Productos Alimenticios Canutos

Representado por Samuel Restrepo

En adelante denominado el Distribuidor

y

HUERTA CO. S.R.L

Bs. As. - República Argentina

Representado por el Sr. David Felipe Ramirez H

Apoderado

\section{ARTÍCULO 1 - DEFINICIONES}

Las partes acuerdan lo siguiente:

Productos Alimenticios Canutos es denominada Fabricante

El Distribuidor denominado la sociedad es Huerta Co S.R.L.

Las partes se denominan en conjunto Huerta Co S.R.L y Productos

Alimenticios Canutos

El territorio es República de Argentina, exclusivamente.

Ambas partes se reconocen mutuamente y tienen la capacidad legal y comercial para el otorgamiento y celebración del presente convenio.

\section{ARTÍCULO 2 - OBJETIVO}

El presente protocolo tiene por finalidad definir los términos y condiciones de distribución de los productos producidos por Productos Alimenticios Canutos en el territorio.

\section{ARTÍCULO 3 - CONDICIONES DE DISTRIBUCIÓN}

Productos Alimenticios Canutos designa al distribuidor concesionario en el territorio para la venta y la distribución de los productos fabricados con marca y calidad "Canutos".

El distribuidor se compromete a :

1- Comprar a Productos Alimenticios Canutos una cantidad de productos que se estipulará en montos FOB / FCA, suficientes para garantizar el abastecimiento de los productos en el territorio. Estas cifras serán anualmente revisadas y constituirán una obligación esencial del contrato.

2- No realizar la fabricación de productos pertenecientes a su propia marca y/o por cuenta de cualquier otra marca, similares o idénticos a los que 
Productos Alimenticios Canutos pretende comercializar por intermedio del distribuidor en el territorio.

\section{ARTÍCULO 4- DURACIÓN}

El presente acuerdo se firma por un período de 3 años y la validez de este contrato tiene vigencia desde la fecha de firma de ambas partes (o de la última firma). Se renueva automáticamente por un período de 2 años, salvo aviso en contrario de una $u$ otra de las partes, debidamente notificado por carta certificada con un preaviso de 3 meses antes de la fecha de vencimiento. En caso de no concretarse ninguna operación durante el primer año de vigencia del contrato, el mismo se considerará rescindido automáticamente.

\section{ARTÍCULO 5- CONDICIONES DE VENTA}

Productos Alimenticios Canutos dará cumplimiento a los pedidos F.O.B. I F.C.A. Buenos Aires, según corresponda embarque marítimo / aéreo-terrestre. Ambas partes acuerdan que los Incoterms utilizados (términos de Comercio Internacional) se considerarán válidos solamente los mencionados por la Cámara de Comercio Internacional, revisión Incoterms 2010.

El lugar de despacho será "F.O.B./ F.C.A. puerto/aeropuerto de Colombia" , punto donde se transfiere la responsabilidad y riesgo de las mercaderías.

El lugar de entrega será a convenir, con un transportista designado por el comprador, con quién y bajo su responsabilidad se efectuará la transferencia del derecho de propiedad de la mercadería exportada, mediante la entrega de documentos de embarque pactados por este medio, vía bancaria u otro medio seguro de envío de documentos.

Los precios podrán ser revisados todos los años, durante el último trimestre del año y el distribuidor será informado de los nuevos precios con un mes de anticipación.

\section{ARTICULO 6 CONDICIONES DE ENTREGA}

Los productos a entregar por Productos Alimenticios Canutos deberán tener como mínimo 6 meses de vencimiento, serán presentados en perfecto estado, listos para la comercialización.

La entrega de los productos será dentro del término de 60 días a contar desde la fecha de la recepción de confirmación del pago por parte de Productos Alimenticios Canutos.

Si durante la negociación del pedido existe algún cambio solicitado por el comprador, el plazo será contado a partir de la fecha del último cambio de pedido posterior a la recepción del anticipo. 
De acuerdo a las condiciones de entrega, se entiende que el precio establecido por el vendedor incluye impuestos, aranceles y tasas relativas a la exportación de los productos, de igual manera el flete y seguro internacional y las eventuales tasas de importación corren por cuenta del comprador.

La pérdida o el deterioro de las mercaderías sobrevenidos después de la transmisión del riesgo al comprador no liberarán a éste de su obligación de pagar el precio, a menos que se deban a un acto u omisión del vendedor.

\section{ARTICULO 7 CONDICIONES DE PAGO}

El distribuidor pagará a Productos Alimenticios Canutos según las siguientes modalidades:

$50 \%$ con el pedido por transferencia swift o cheque debidamente acreditado. Saldo con Letra de Cambio a 45 días.

El monto de compra acordado entre las partes no podrá ser inferior a los U\$S 50.000 anuales, en el transcurso del primer año de obtenidos los registros sanitarios. (Son Dólares Estadounidenses Cien mil). Para el segundo año será de u\$s 70.000.- y para el tercer año de u\$s 100.000.

La aceptación de la oferta surtirá efecto en el momento en que la indicación de asentimiento llegue al oferente, y no surtirá efecto si la indicación de asentimiento no llega al oferente dentro del plazo que éste haya fijado o, si no se ha fijado plazo, dentro de un plazo razonable, habida cuenta de las circunstancias de la transacción y, en particular, de la rapidez de los medios de comunicación empleados por el oferente.

\section{ARTÍCULO 8 - DOCUMENTOS DE EMBARQUE}

En cada envío, el fabricante se compromete a enviar los siguientes documentos emitidos por su cuenta y riesgo:

Factura comercial

Lista de empaque

En caso de que el distribuidor lo considere necesario, o por exigencias de ingreso en el territorio, Productos Alimenticios Canutos podrá gestionar:

Cerificado de análisis

Cerificado de origen

En estos casos, el comprador deberá indicar alguna cláusula especial a ser incluida en la confección de estos documentos.

Respecto del conocimiento de embarque, (B/L) guía aérea, (A.W.B.) u otro documento de embarque, de acuerdo al medio de transporte elegido y acordado entre las partes, Productos Alimenticios Canutos pondrá a 
disposición del mismo la mercadería pagando los gastos y asumiendo responsabilidades hasta el ingreso de la mercadería a bordo, la emisión del documento de transporte estará a cargo del transportista, y la liberación de la mercadería para la importación en destino será a cargo, responsabilidad y costo exclusivamente del comprador, no asumiendo Productos Alimenticios Canutos responsabilidad alguna en esa gestión.

En caso de necesitar alguna condición especial en la contratación del flete internacional, esta debe ser pactada por el comprador directamente con el transportista y comunicada fehacientemente a Productos Alimenticios Canutos y si el comprador lo solicita, Productos Alimenticios Canutos podrá contratar bajo exclusiva responsabilidad, riesgo y costo del importador algún transportista en el mercado local.

Si en el mercado del importador es necesario la obtención de licencias de importación, éstas deberán ser tramitadas por el comprador y su no obtención en tiempo y forma no puede ser tomado por el comprador como motivo de incumplimiento de sus obligaciones de despacho de importación.

En caso de ser necesaria la obtención de certificados o presencia de entidades relativas a cuestiones de certificación de preembarque en origen, esto debe ser comunicado con la suficiente antelación a Productos Alimenticios Canutos, corriendo los gastos de intervención por parte del Importador.

\section{ARTÍCULO 9 - PROMOCIÓN}

El distribuidor se compromete a afrontar los gastos de promoción y desarrollo de las ventas de productos en el territorio y a velar por los intereses y la imagen de marca de Productos Alimenticios Canutos.

Todo material de promoción preparado por el distribuidor será sometido a Revisión para su aprobación y estudio.

El distribuidor, el 1 de junio de cada año ( o antes de esta fecha), presentará a Productos Alimenticios Canutos en el territorio y por producto sus previsiones de venta. Esto ayudará a Productos Alimenticios Canutos a planificar sus producciones.

\section{ARTÍCULO 10 - COMPETENCIA}

Se prohíbe al distribuidor obstaculizar directa o indirectamente, por cualquier circunstancia que fuere, la venta de los productos en el territorio, se prohíbe especialmente vender productos que directa 0 indirectamente fueran competitivos, sin tener el consentimiento previo por escrito de Productos Alimenticios Canutos.

ARTÍCULO 11 - REGISTRO 
El distribuidor se compromete a colaborar de la manera mas conveniente poniendo sus medios a disposición de Productos Alimenticios Canutos para todo tipo de negociaciones, la redacción de textos apropiados y para toda acción necesaria para la obtención de registros indispensables por ante las autoridades competentes, como así también toda modificación que fuera necesaria para la evolución de la legislación o del mercado y ello para el conjunto de los productos vendidos o por vender en el territorio.

Dichos registros serán emitidos por la dirección nacional de registro sanitaria a nombre de Productos Alimenticios Canutos

El costo de análisis, sellados y otorgamiento de registro para la habilitación y venta de cada producto serán a cargo de Huerta Co S.R.L., reintegrando Productos Alimenticios Canutos el costo del registro en mercadería igual a la registrada. Todo ello una vez acreditada por el distribuidor en forma fehaciente la referida inscripción y documentado el costo total de la misma.

En caso de necesitar el distribuidor etiquetas, envase o embalajes con escrituras o leyendas especiales, esto deberá ser informado con antelación por el distribuidor, mediante escrito especificando las características de la leyenda o escritura.

\section{ARTÍCULO 12 - CONFIDENCIALIDAD}

El distribuidor admite que las informaciones que les serán confiadas en el marco de su colaboración con Productos Alimenticios Canutos son confidenciales.

El distribuidor acepta el total control de las informaciones que le serán comunicadas en el marco de este acuerdo.

En consecuencia, el distribuidor se obliga a no divulgar a terceros, personas físicas o morales, las informaciones así obtenidas de manera directa o indirecta.

Se conviene expresamente entre las partes que la presente cláusula continuara aún luego de la rescisión del contrato de distribución, cualesquiera sea la causa o a su término.

\section{ARTÍCULO 13 - INFORMACIÓN COMERCIAL}

Las partes admiten la importancia de la promoción de los productos en el territorio.

En consecuencia, el distribuidor se obliga a suministrar a Productos Alimenticios Canutos todas las informaciones necesarias para el desarrollo de negocios en el territorio.

ARTÍCULO 14 - LIMITACIÓN DE LA REPRESENTACIÓN 
Ninguna cláusula de este contrato podrá ser interpretada como otorgamiento al distribuidor de algún mandato o poder de representación más allá de lo expresamente establecido en el presente contrato.

En consecuencia, el distribuidor no podrá comprometer a Productos Alimenticios Canutos frente a terceros y cualquier acto que pudiera realizar a nombre de Productos Alimenticios Canutos sería nulo y se tendrá por no escrito, comprometiendo solamente la responsabilidad del distribuidor.

Ninguna alteración podrá ser efectuada en este contrato sin el acuerdo escrito de ambas partes.

Productos Alimenticios Canutos no será responsable solidario con el distribuidor en caso de que este cometa actos comerciales o de otra índole que pudiesen perjudicar su imagen comercial en la región.

\section{ARTÍ́CULO 15 - RESCISIÓN}

En el caso de que una de las partes incurriera en algún incumplimiento y no lo subsanara en un plazo de 30 días, luego de haber sido constituido en mora por carta certificada o telex, la otra parte podrá rescindir el contrato de pleno derecho también por carta certificada.

La rescisión entrará en vigencia a partir de la notificación de la misma, sin perjuicio de cualquier acción por daños y perjuicios.

\section{ARTÍCULO 16 - RESPONSABILIDAD}

La responsabilidad de Productos Alimenticios Canutos frente al distribuidor y a los terceros ante cualquier conflicto o proceso, está estrictamente limitada a la calidad de los productos puestos en Colombia (Puesto en Aeropuerto) y a la validez de las patentes de fabricación de República Argentina, y ante cualquier eventual reclamo, el mismo no podrá, en ningún caso, exceder el precio de la mercadería objeto de reclamo.

Con respecto al transpaso de responsabilidad y riesgo en relación a las mercaderías envíadas por Productos Alimenticios Canutos, ambas partes aceptan los límites establecidos por el Incoterm que define la operación.

Se acuerda expresamente que Productos Alimenticios Canutos en ningún caso podrá ser responsable, frente a terceros y/o al distribuidor, por todos los daños indirectos tales como la pérdida de ganancia o de explotación.

El distribuidor, deberá respetar sus obligaciones legales en materia de póliza de seguros y hacer que Productos Alimenticios Canutos pueda ejercer sus derechos en las mejores condiciones.

ARTÍCULO 17 - FUERZA MAYOR 
Productos Alimenticios Canutos nunca será considerada responsable por las pérdidas y daños ocurridos como consecuencia de accidentes, escasez de materias primas o materiales, huelgas, catástrofes naturales, casos de fuerza mayor, modificación de las reglamentaciones internacionales o nacionales, y generalmente cualquier causa que escapara a su control.

En este caso, se procederá a una revisión de las cuotas correspondiente a los pedidos no entregados.

En el caso de que el contrato fuera suspendido por fuerza mayor, por un período que supera los 180 días, las partes, podrán decidir de común acuerdo rescindir el presente contrato en las condiciones del artículo 15, sin culpa de las partes y sin derecho alguno a indemnizaciones.

\section{ARTÍCULO 18 - SANEAMIENTO JUDICIAL}

Productos Alimenticios Canutos podrá rescindir el contrato con efecto inmediato, en el caso de que el distribuidor solicitara su quiebra o fuera declarado en quiebra, hubiera caído en insolvencia, procediera a un abandono de su activo a favor de sus acreedores, fuera objeto de una disolución voluntaria o judicial, entrara en saneamiento judicial o en liquidación o se produjera una modificación del control de su capital.

\section{ARTÍ́CULO 19 - PAGO}

La suspensión de este contrato, cualesquiera fuera la causa, no libera al distribuidor de su obligación de pago hacia Productos Alimenticios Canutos, en virtud de dicho contrato.

\section{ARTÍCULO 20 - CESIÓN DEL CONTRATO}

Productos Alimenticios Canutos, podrá sin formalidad alguna ceder total 0 parcialmente todos sus derechos, facultades y obligaciones, a cualquier sociedad filial o afiliada.

Fuera de esta situación, ninguna parte podrá transferir sus derechos, sin el consentimiento previo y escrito de la otra parte.

\section{ARTICULO 21 - SEGURO}

Sin perjuicio de las condiciones particulares, los productos serán entregados de acuerdo a lo expuesto en los artículos 5 y 6, por lo que Productos Alimenticios Canutos nunca podrá ser declarado responsable por daños ocurridos.

El distribuidor se compromete a todas las precauciones necesarias para asegurar las mercaderías más alla de este punto.

Productos Alimenticios Canutos cumple su obligación principal cuando haga entrega de la mercadería conforme al contrato de venta y remita la documentación de envío al distribuidor o a su agente. 


\section{ARTÍCULO 22 - LEY APLICABLE Y COMPETENCIA}

En caso de litigio en la interpretación, ejecución o rescisión de este contrato, las partes reconocen la competencia exclusiva de los Tribunales Federales de la República Argentina. La ley aplicada es la Ley Argentina.

\section{ARTÍ́CULO 23 - GENERAL}

Este acuerdo constituye la integralidad de este contrato entre las partes, no podrá ser modificado salvo por acuerdo escrito.

\section{ARTÍCULO 24 - DOMICILIOS}

A todos los efectos legales, judiciales y extrajudiciales que se deriven del presente contrato, Productos Alimenticios Canutos fija domicilio en el barrio Manila de Medellín Colombia y el distribuidor lo hace en la calle , Lavalle 774 Oficina 8 c del Barrio San Nicolás de Capital Federal de Buenos Aires.

En estos domicilios serán válidas todas las notificaciones y/o citaciones que deban cursarse las partes.

Celebrado en Buenos Aires, el 15 de Enero de 2013

David Felipe Ramírez Hernández

DNI. 94.711.563

Apoderado

Apoderado 


\section{ANEXO 3 CICLO DEL PRODUCTO}

\section{Extensión Ciclo de vida del Producto}

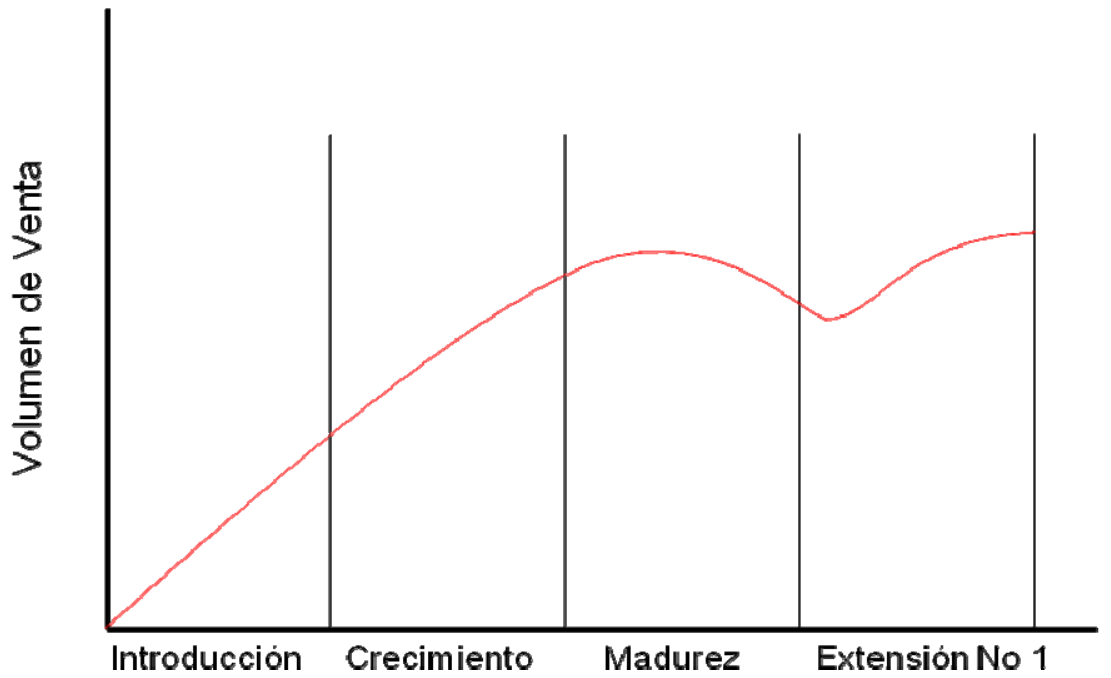

ANEXO 4 MODELO DEL PELLET CON 30 CAJAS DE 22 UNIDADES

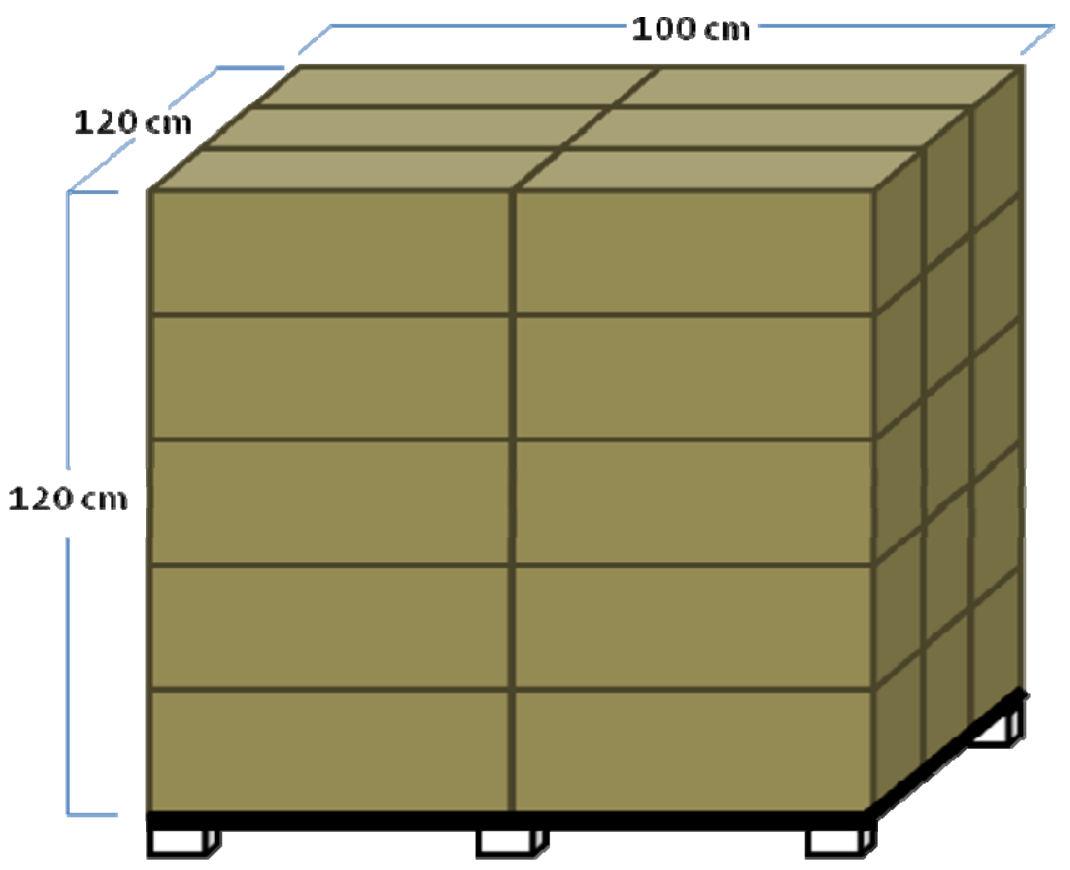




\section{Cuerpo 1}
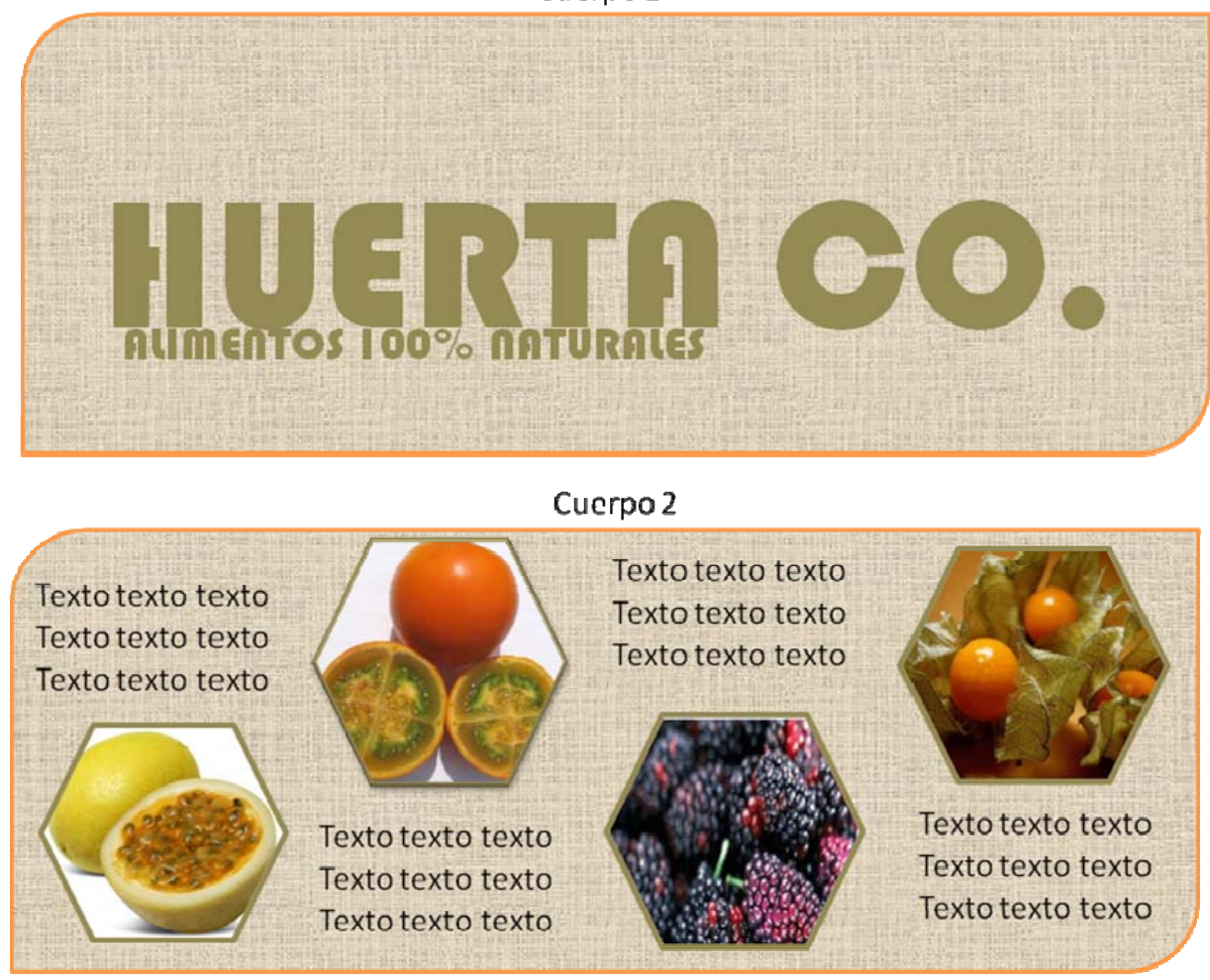

Cucrpo3

\section{RECETAS ARTESAMALES OUE SIGUEA DELEITANDO}

Texto Texto Texto

Texto Texto Texto

Texto Texto Texto

Texto Texto Texto
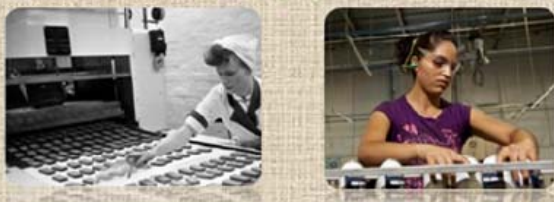

Texto Texto Texto Texto Texto Texto Texto Texto Texto

Texto Texto Texto Texto Texto Texto Texto Texto Texto Texto Texto Texto

Texto Texto Texto Texto Texto Texto Texto Texto Texto Texto Texto Texto Texto Texto Texto Texto Texto Texto

Texto Texto Texto Texto Texto Texto

\section{Cucrpo 4}

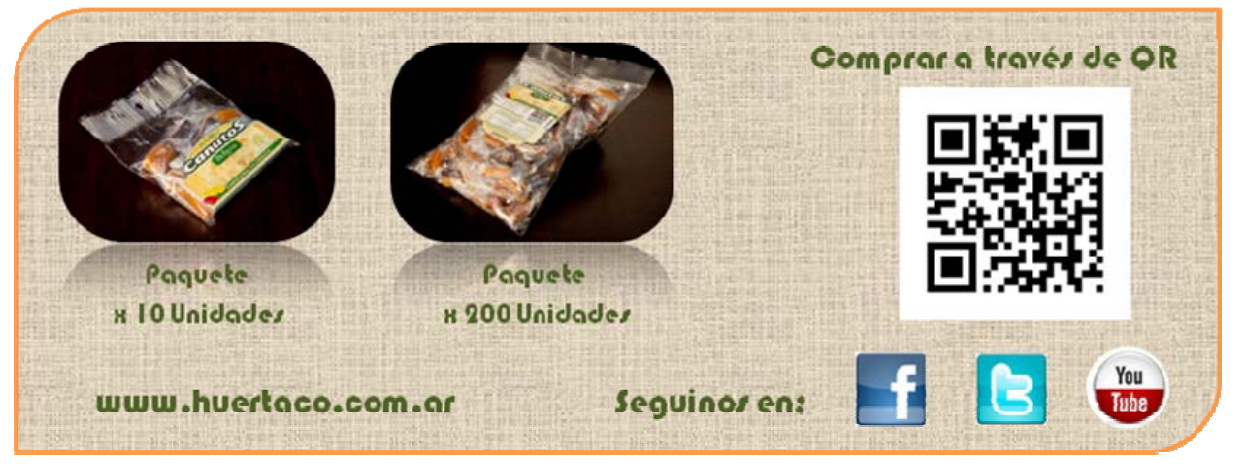




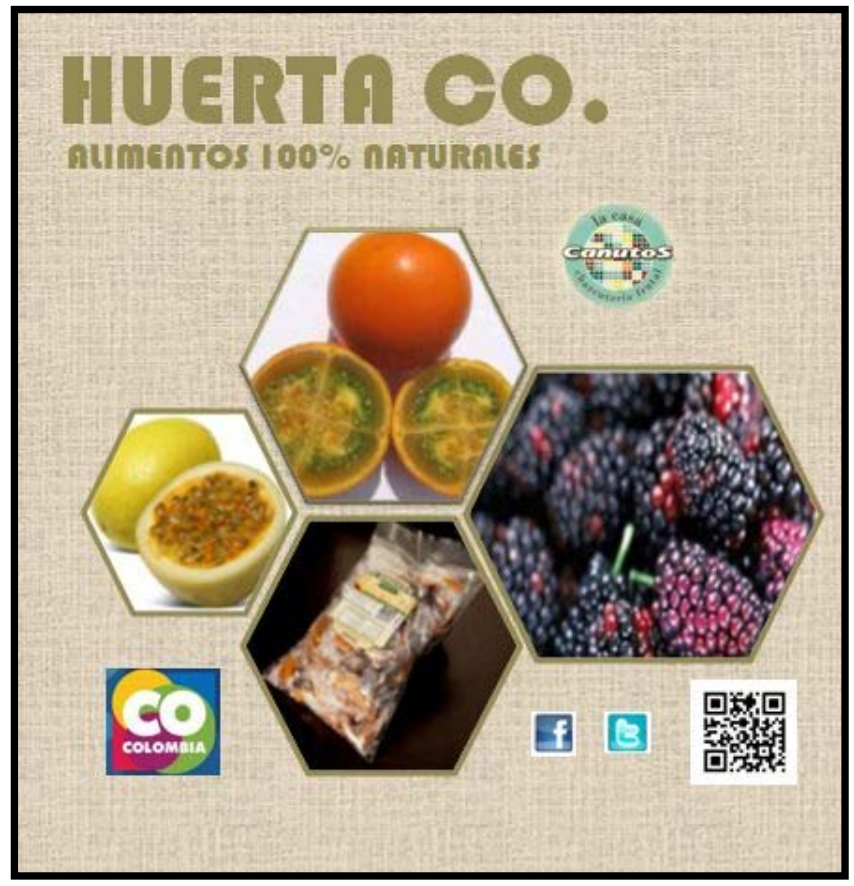

ANEXO No 8 TARJETAS DE PRESENTACION

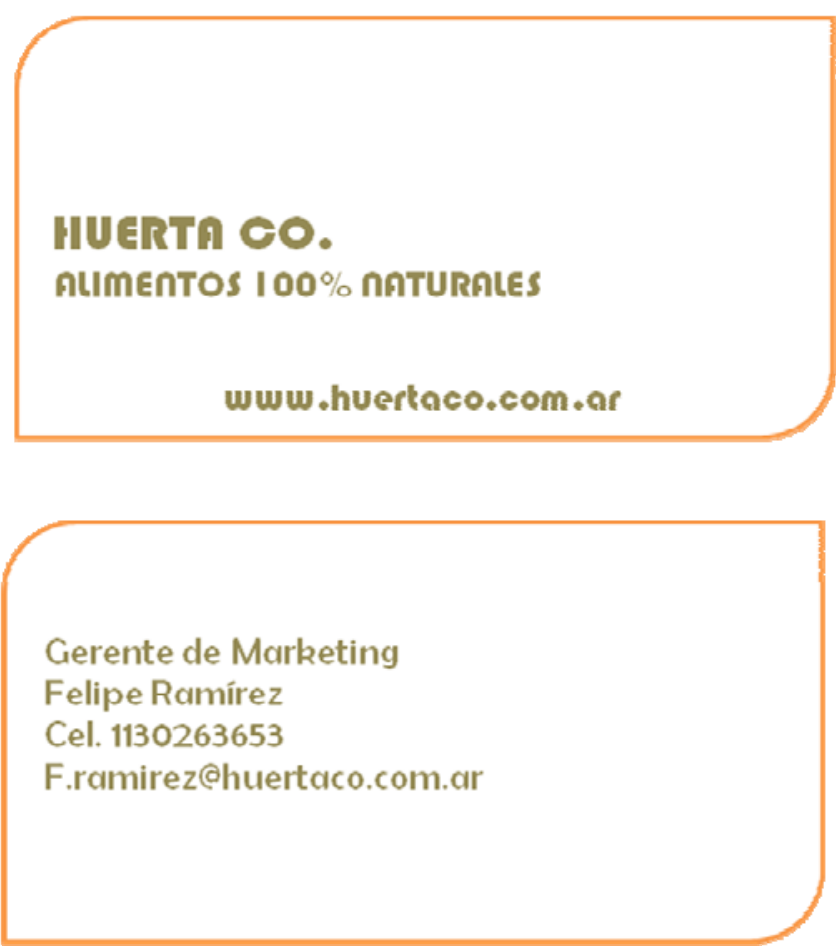




\section{ACLARACION}

Yo David Felipe Ramírez Hernández aseguro que esta tesis fue elaborada por mi, que no utilice ningún otro material que no haya dado a conocer en las referencias y que no utilice frases o párrafos de otros autores, que este trabajo de tesis nunca ha sido presentado ante un comité de evaluación de tesis y que no trasgreda derechos de terceros.

Firma

Aclaración

Numero de Documento 